\title{
The critical care management of spontaneous intracranial hemorrhage: a contemporary review
}

\author{
Airton Leonardo de Oliveira Manoel ${ }^{1,2,3^{*}}$ (D), Alberto Goffi ${ }^{4,5}$, Fernando Godinho Zampieri, ${ }^{6,7}$, David Turkel-Parrella ${ }^{1}$, \\ Abhijit Duggal ${ }^{8,9}$, Thomas R. Marotta ${ }^{1}$, R. Loch Macdonald ${ }^{10}$ and Simon Abrahamson ${ }^{2,11}$
}

\begin{abstract}
Spontaneous intracerebral hemorrhage $(\mathrm{ICH})$, defined as nontraumatic bleeding into the brain parenchyma, is the second most common subtype of stroke, with 5.3 million cases and over 3 million deaths reported worldwide in 2010. Case fatality is extremely high (reaching approximately $60 \%$ at 1 year post event). Only $20 \%$ of patients who survive are independent within 6 months. Factors such as chronic hypertension, cerebral amyloid angiopathy, and anticoagulation are commonly associated with $\mathrm{ICH}$. Chronic arterial hypertension represents the major risk factor for bleeding. The incidence of hypertension-related ICH is decreasing in some regions due to improvements in the treatment of chronic hypertension. Anticoagulant-related ICH (vitamin $\mathrm{K}$ antagonists and the newer oral anticoagulant drugs) represents an increasing cause of $\mathrm{ICH}$, currently accounting for more than $15 \%$ of all cases. Although questions regarding the optimal medical and surgical management of $\mathrm{ICH}$ still remain, recent clinical trials examining hemostatic therapy, blood pressure control, and hematoma evacuation have advanced our understanding of $\mathrm{ICH}$ management. Timely and aggressive management in the acute phase may mitigate secondary brain injury. The initial management should include: initial medical stabilization; rapid, accurate neuroimaging to establish the diagnosis and elucidate an etiology; standardized neurologic assessment to determine baseline severity; prevention of hematoma expansion (blood pressure management and reversal of coagulopathy); consideration of early surgical intervention; and prevention of secondary brain injury. This review aims to provide a clinical approach for the practicing clinician.
\end{abstract}

\section{Background}

Spontaneous intracerebral hemorrhage (ICH) is defined as nontraumatic bleeding into the brain parenchyma $[1,2]$, which can extend into the ventricles and into the subarachnoid space [3]. ICH is the second most common subtype of stroke [3], accounting for $10-50 \%$ of all cases $[4,5]$, depending on the population, race, and region studied [6]. According to the "Global Burden of Diseases, Injuries, and Risk Factors" report, there were 5.3 million cases and over 3.0 million deaths secondary to ICH worldwide in 2010 [6, 7]. The case-fatality rate ranges

\footnotetext{
* Correspondence: airtonleo.manoel@gmail.com

'Department of Medical Imaging, Interventional Neuroradiology, St. Michael's Hospital-University of Toronto, 30 Bond Street, Toronto, ON M5B 1W8, Canada

${ }^{2}$ Department of Critical Care Medicine, Trauma \& Neurosurgical Intensive Care Unit, St. Michael's Hospital-University of Toronto, Toronto, ON, Canada Full list of author information is available at the end of the article
}

from $35 \%$ at 7 days to $59 \%$ at 1 year [8-10]. Half of fatal cases occur in the first 48 hours after presentation [11, 12]. Survivors are often left with severe disability [9], with less than $40 \%$ of patients regaining functional independence [3]. The epidemiology of ICH may change in the future with better control of risk factors such as hypertension [13], but the use of newer anticoagulation therapies may influence the acute management and possibly prognosis of the disease $[14,15]$.

ICH has been traditionally described as the subtype of stroke with the poorest prognosis [10]. However, recent observational reports suggested that self-fulfilling prognostic pessimism may lead to withdrawal of life support in patients who otherwise may have had an acceptable clinical outcome if managed aggressively [11]. Part of the pessimism surrounding the prognostication of hemorrhagic stroke is hypothesized to be a tendency not to consider 
factors such as age, previous comorbidities, etiology of the bleeding, and socioeconomic factors [12], which are known to affect outcome [16].

\section{Etiology and risk factors}

The most important modifiable risk factor in spontaneous ICH is chronic arterial hypertension [17]. Deep perforator arteries in the pons, midbrain, thalamus, basal ganglia, and deep cerebellar nuclei, chronically damaged by hypertension, are the most common locations for hypertensive bleeding $[18,19]$. Chronic hypertension is present in 50 $70 \%$ of patients who develop ICH [20]. Patients with a systolic blood pressure (SBP) $\geq 160 \mathrm{mmHg}$ or a diastolic blood pressure $\geq 110 \mathrm{mmHg}$ have a 5.5 (95\% CI 3.0-10.0) times increased rate of $\mathrm{ICH}$, compared with normotensive patients [21]. Besides hypertension, cerebrovascular amyloid deposition (i.e., cerebral amyloid angiopathy) is associated with ICH in older patients [22]. Intracranial hemorrhage associated with cerebral amyloid angiopathy seldom occurs in subjects younger than 60 years of age. The incidence significantly increases thereafter, and is almost always associated with a lobar hemorrhage [23]. Coagulopathies (i.e., the use of antithrombotic or thrombolytic agents, congenital or acquired factor deficiencies) and systemic diseases, such as thrombocytopenia, are possible causes of $\mathrm{ICH}$. The use of oral anticoagulants, especially vitamin $\mathrm{K}$ inhibitors (i.e., warfarin), has increased coagulopathy-associated ICH in recent years, accounting for more than $15 \%$ of all cases $[14,24]$.
Psychosocial, ethnic, and economic factors play a role in the prevalence of cerebral hemorrhage, with $\mathrm{ICH}$ being twice as common in low-income and middle-income countries compared with high-income countries [2, 19, 25]. Other identified risk factors for $\mathrm{ICH}$ include age (i.e., each decade from 50 years of age is associated with a 2 -fold increase in the incidence of $\mathrm{ICH}$ ) and an elevated alcohol intake [20].

Etiologies of ICH to always consider include: intracranial aneurysms (typically presenting as subarachnoid hemorrhage); arteriovenous malformations ( $\mathrm{ICH}$ is the first presentation of AVMs in $60 \%$ of cases); cerebral venous sinus thrombosis and venous infarction; brain tumors ( $<5 \%$ of all ICH cases) including cerebral metastasis (e.g., lung cancer, melanoma, renal cell carcinoma, thyroid carcinoma, and choriocarcinoma) and primary CNS tumors (e.g., glioblastoma multiforme and oligodendrogliomas); and drugs of abuse (e.g., cocaine, amphetamines). Because of the differing etiologies of $\mathrm{ICH}$, a rapid and accurate diagnosis of the underlying etiology of $\mathrm{ICH}$ is essential to direct appropriate management strategies.

\section{Initial evaluation and clinical stabilization}

According to the AHA/ASA guidelines [9] and the Emergency Neurological Life Support protocols [26], spontaneous intracranial hemorrhage is a medical emergency and should be managed accordingly. The initial management should focus on the following principles (Fig. 1):

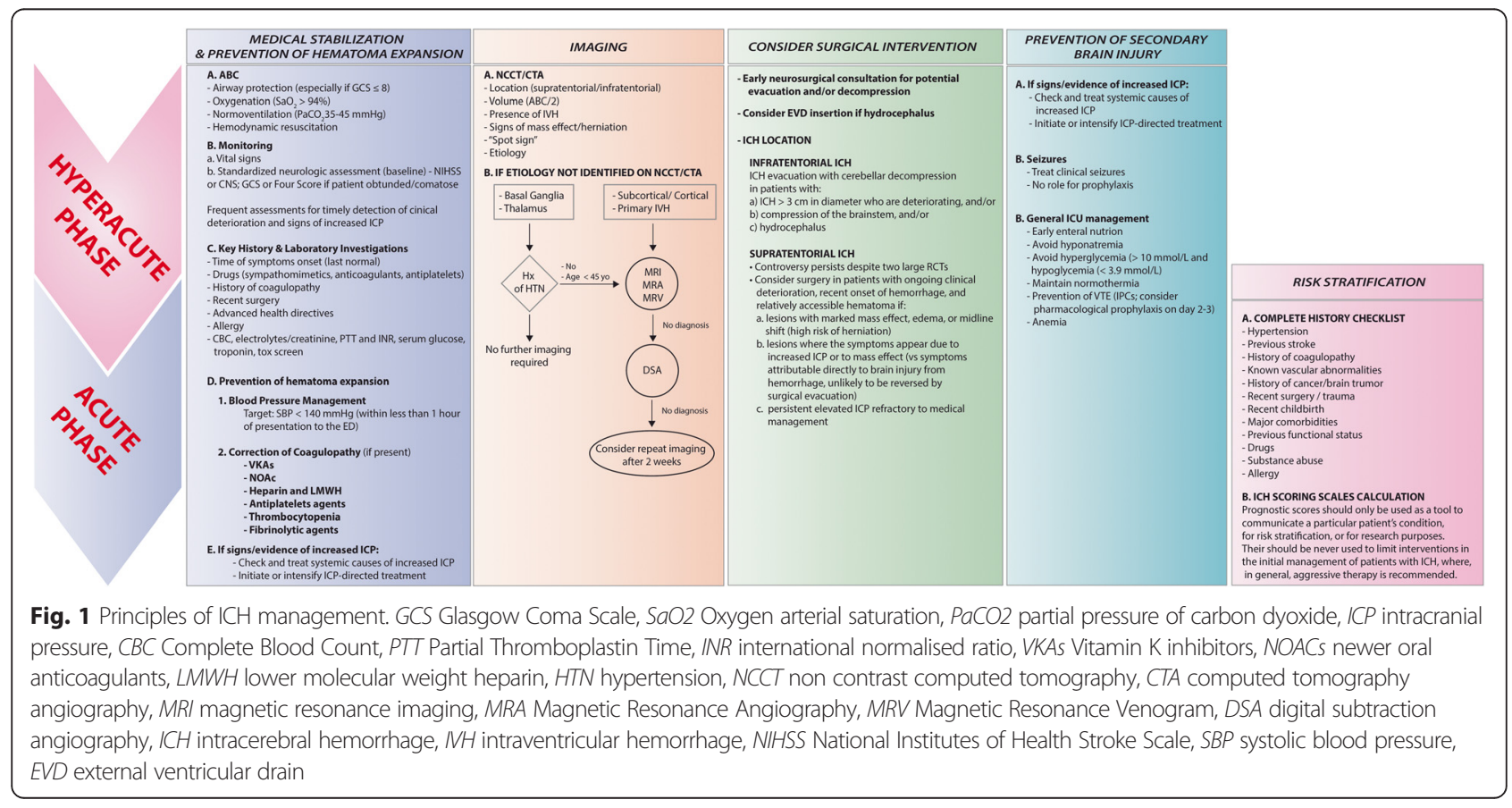


1. ABC's. Initial assessment and stabilization of airway patency, breathing, and circulation.

2. Neuroimaging. Once clinical stability is achieved, an urgent imaging study for rapid and accurate diagnosis should be performed.

3. Standardized neurologic assessment to determine baseline severity. The National Institutes of Health Stroke Scale (NIHSS), if the patient is awake or drowsy, or the Glasgow Coma Scale (GCS), if the patient is obtunded or comatose, should be performed and clearly documented.

4. Blood pressure management, reversal of coagulopathy, and evaluation of the need for early surgical intervention.

5. Frequent neurological examinations, at least every hour $[27,28]$, to detect early clinical deterioration and signs of increased intracranial pressure (ICP) should be part of the routine initial management algorithm.

\section{Emergency radiologic diagnosis}

The clinical presentation of ICH does not differ from acute ischemic stroke, with the sudden onset of a focal neurological deficit. However, certain clinical findings significantly increase the probability of intracranial hemorrhage, such as coma, neck stiffness, seizures accompanying the neurologic deficit, diastolic blood pressure $>110 \mathrm{mmHg}$, vomiting, and headache. Cervical bruit and prior transient ischemic attack decrease the probability of hemorrhagic stroke. However, no single clinical finding or combination of clinical findings can be considered absolutely diagnostic [29]. Neuroimaging is therefore crucial in establishing the diagnosis, and also for consideration of the underlying etiology. Current guidelines recommend either noncontrast computed tomography $(\mathrm{CT})$ or magnetic resonance imaging (MRI) as the initial imaging test (Class I; Level of Evidence A) [9]. CT is usually the first-line modality given its wide availability and rapidity. Noncontrast CT is highly sensitive and specific for acute blood [9]. Magnetic resonance (gradient echo or T2 susceptibility-weighted sequences) is as sensitive as noncontrast $\mathrm{CT}$ in detecting acute blood, and is more sensitive in detecting previous hemorrhage. The main drawbacks of MRI use in the acute setting are cost and availability [30].

Computed tomography angiography (CTA) or contrast-enhanced CT is also commonly employed in the acute setting, combined with noncontrast $\mathrm{CT}$, as a strategy to detect active contrast extravasation into the hematoma (spot sign-discussed later). Additionally, once ICH is confirmed, additional imaging (e.g., CTA, magnetic resonance angiography, or digital subtraction angiography (DSA)) is recommended to search for the underlying etiology (Additional file 1: Table S1), such as vascular malformations and brain tumors (Class IIa; Level of Evidence B)
[31, 32]. If cerebral venous sinus thrombosis is suspected because of radiological findings such as unusual hematoma location, relative increased edema volume, or abnormal signal in the cerebral sinuses, CT venography or magnetic resonance venography should be performed [33].

In hypertensive patients older than 65 years with a wellcircumscribed hematoma located in the basal ganglia or thalamus, the yield of such studies is low $(2-3 \%)$ and the decision not to proceed with further diagnostics tests may be reasonable [31, 32]. In young non-hypertensive patients, the following findings may warrant additional work-up: presence of isolated intraventricular hemorrhage (IVH) or subarachnoid hemorrhage, noncircular hematoma shape, disproportionately excessive edema, lobar location, identification of space-occupying lesion, enlarged vessels or calcifications along the margins of the $\mathrm{ICH}$, and hyperattenuation within a dural venous sinus or cortical vein along the presumed venous drainage path. DSA remains the gold standard for identifying underlying vascular lesions (e.g., cerebral aneurysms, arteriovenous malformations), but CTA has shown an accuracy of $89-100 \%$ for determining secondary causes of ICH $[34,35]$. A recent study comparing CTA with DSA in nonhypertensive patients younger than 45 years old demonstrated excellent negative and positive predictive values of CTA and CT venography $(97.3 \%$ and $100 \%$, respectively) to establish or exclude vascular causes of ICH [36].

\section{$\mathrm{ICH}$ volume, IVH, and hematoma location}

The volume of blood on the initial noncontrast CT image has a strong independent association with outcome. A hematoma volume of $30 \mathrm{ml}$ represents a cutoff point for increased mortality $[37,38]$ and worse functional outcome [37]. The ICH volume can be estimated using the $\mathrm{ABC} / 2$ formula [39], "where $\mathrm{A}$ is the greatest hemorrhage diameter by CT, B is the diameter $90^{\circ}$ to $\mathrm{A}$, and $\mathrm{C}$ is the approximate number of $\mathrm{CT}$ slices with hemorrhage multiplied by the slice thickness" [40]. Additionally, when the hematoma volume is combined with the initial level of consciousness assessed by the GCS, it can accurately predict 30-day mortality [37]. Patients with an ICH volume of $\geq 60 \mathrm{ml}$ on the initial CT image and GCS $\leq 8$ have a predicted 30-day mortality $>90 \%$, compared with a mortality of $<20 \%$ for patients with hematoma volume $<30 \mathrm{ml}$ and $\mathrm{GCS} \geq 9$ [37].

Both the presence and ongoing expansion of IVH are powerful and independent predictors of functional outcomes after ICH [41]. IVH is present in approximately $45 \%$ of patients with spontaneous ICH. IVH is associated with a lower probability of favorable outcome compared with absence of IVH (15\% vs $31 \%, p<0.00001)$ [42]. An increase of more than $2 \mathrm{ml}$ in IVH volume in the first 24 hours has been shown to be associated with an odds 
ratio (OR) for a poor outcome of 4.2 (95 \% CI 1.06-16.63, $p=0.0405)$ [43].

Hematoma location is another important factor that affects outcome and treatment [26]. The most common locations of hypertensive $\mathrm{ICH}$ are the basal ganglia (caudate nucleus and putamen), thalamus, deep cerebellar nuclei, midbrain, or pons (Fig. 2). Lobar hemorrhages are often associated with structural changes such as cerebral amyloid angiopathy, arteriovenous malformations, or brain tumors.

\section{Early neurological deterioration and hematoma expansion}

ICH patients are at risk for early neurological deterioration, which is usually secondary to early hematoma expansion or the development of acute hydrocephalus secondary to IVH $[44,45]$. The definition of early neurological deterioration varies across studies, but it is usually described as worsening from the initial neurological examination (e.g., a change in the initial GCS or NIHSS scores) or progression to death [46]. Early neurological deterioration occurs in up to $40 \%$ of patients within 48 hours, and is associated with poorer long-term prognosis [46]. Mayer et al. [44] described a prospective cohort of 46 noncomatose patients
$(\mathrm{GCS} \geq 8)$ with $\mathrm{ICH}$. Fifteen patients $(33 \%)$ developed neurological deterioration, the majority $(8 / 15,53 \%)$ during the first day of hospitalization. Patients with neurological deterioration had larger hematoma volume (mean volume: $45 \mathrm{ml}$ vs $16 \mathrm{ml}, p<0.01$ ) and mass effect on initial CT (60\% vs $19 \%, p<0.01$ ). The 30 -day mortality was $47 \%$ in patients with neurological deterioration compared with $3 \%$ in those patients who did not acutely worsen $(p=0.001)$. While the two most important predictors on hospital admission of early neurological deterioration are the hematoma volume and the presence of IVH, other factors such as glucose concentration, fibrinogen levels, and elevated SBP have been described [46].

Hematoma expansion is a major determinant of early neurological deterioration, poor outcome, and death [47-49]. Brott et al. [45] found that $26 \%$ of ICH patients developed substantial hemorrhage growth (defined as $\geq 33 \%$ increase from baseline hematoma volume) between baseline and 1-hour CT scans (i.e., within 4 hours of symptom onset). Additionally $12 \%$ of the patients developed hematoma growth between the 1-hour and 20 -hour CT scans. Hemorrhage growth was significantly associated with early neurological deterioration. Hematoma expansion is an independent determinant of poor outcome

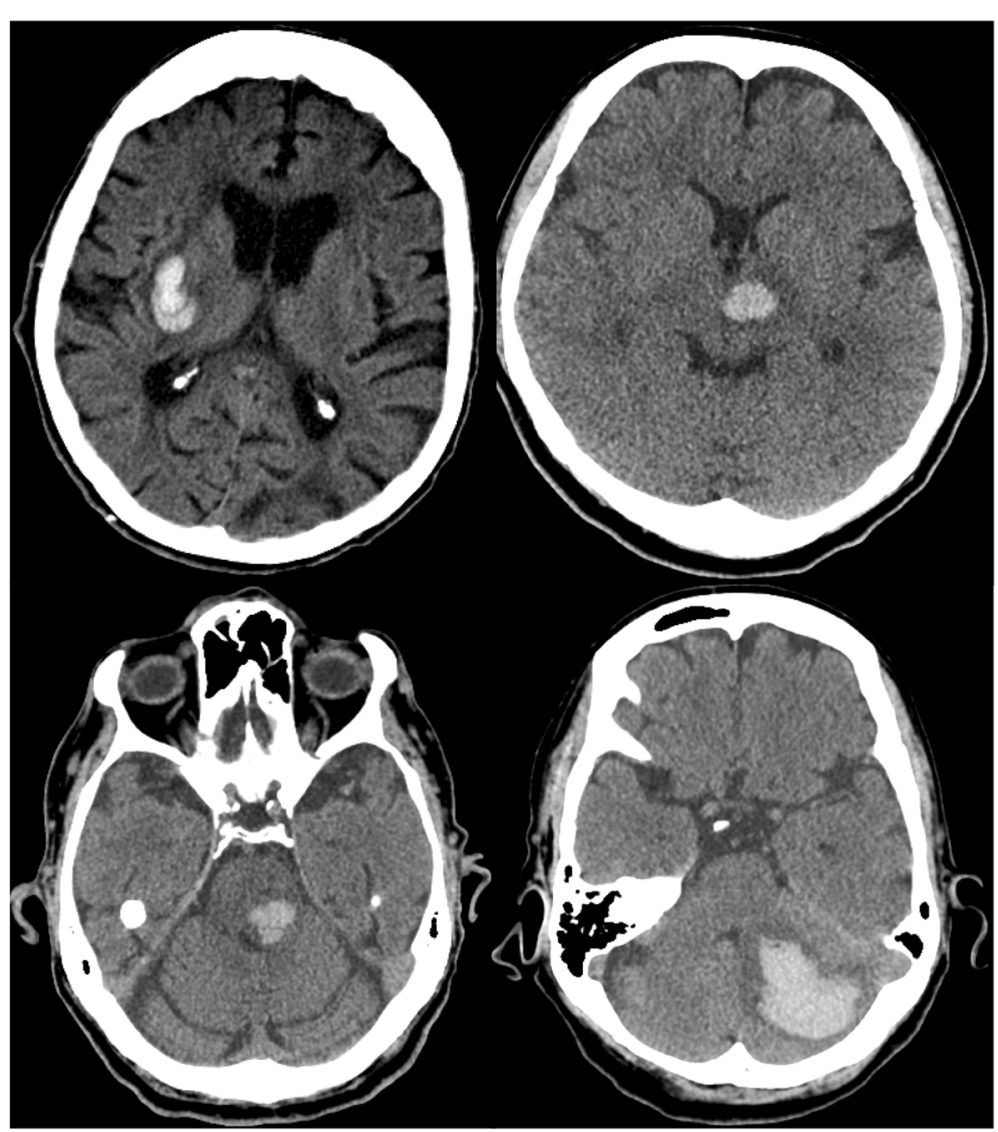

Fig. 2 Deep intracranial hemorrhage. Common locations of hypertensive hemorrhage (clockwise: putamen, thalamus, cerebellum, and pons) 
and mortality [47], regardless of hematoma expansion definition [48]. The early occurrence of hematoma growth and subsequent neurological deterioration highlights the importance of frequent neurological examinations and early repeat CT scanning, which can alter medical patient care or may trigger surgical interventions [28].

\section{Prediction of hematoma expansion}

Because hematoma expansion is a major determinant of mortality and functional outcome, it could be potentially beneficial to identify those patients at highest risk of hematoma expansion. Prediction scores have been published to predict hematoma expansion in $\mathrm{ICH}$ (Additional file 1: Table S5) [50-52]. Prediction scores share several common factors: shorter time from $\mathrm{ICH}$ onset to CT; warfarin use; and evidence of spot sign on CTA (see later). The risk of hematoma expansion varies from $3.4-7.1 \%$ in patients with no risk factors [50-52] to 70$85.5 \%$ in patients obtaining the maximum score [50-52].

(1) Time from ICH onset to CT. As discussed previously, hematoma expansion tends to occur early in the course of $\mathrm{ICH}$ [45], with the majority (26\%) of significant hematoma growth happening between baseline and 1-hour CT scans, compared with only $12 \%$ between 1-hour and 20-hour CT scans. It is not surprising that a shorter time between $\mathrm{ICH}$ onset and CT scan would appear to be a predictor of hematoma expansion, because CT scans performed more than 6 hours after ICH onset would probably miss hematoma growth that may have already occurred [53].

(2) Patients on warfarin (or with International Normalized Ratio (INR) > 1.5) have an adjusted OR of 4.04

(95 \% CI 1.85-9.13) for hematoma expansion compared with patients not on warfarin. Warfarin-related $\mathrm{ICH}$ is discussed in the anticoagulant-associated $\mathrm{ICH}(\mathrm{AAICH})$ section.

(3) “Spot sign” (Fig. 3). Initially described as contrast extravasation on CTA [54-56], the term spot sign has evolved to encompass foci of enhancement within the hematoma on CTA [57]. Presently, the term "contrast extravasation" should only be used to describe the presence of contrast within the hematoma on post-contrast CT $[58,59]$. The identification of the spot sign (Fig. 3), and its sensitivity in predicting hematoma expansion, is dependent on several technical aspects of imaging acquisition, such as the concentration of the contrast agent, the speed of scanners, and, importantly, the timing between the contrast injection and the image acquisition [60, 61]. The spot sign can be divided into the early spot sign and the delayed spot sign. The early spot sign is detected on the first-pass CTA, usually acquired in the arterial phase within 30 seconds after contrast injection. The delayed spot sign is detected on the second-pass CTA, or post-contrast CT. The secondpass CTA images (or venous phase of CTA), acquired 40 seconds to 3 minutes after contrast injection, increase the yield of identifying a spot sign if not visualized on the first-pass CTA [58, 59, 62-64]. In a systematic review and meta-analysis [53] of 14 studies of first-pass CTA, the spot sign had a sensitivity of $53 \%$ (95\% CI $49-57 \%$ ), a specificity of $88 \%$ (95\% CI 86-89\%), a positive likelihood ratio of 4.70 (95 \% CI 3.28-6.74), and a negative likelihood ratio of 0.44 (95\% CI 0.34-0.58) for predicting hematoma expansion. When a first-pass CTA was combined with a post-contrast CT (data available for three studies) better accuracy was achieved, with a sensitivity of $92 \%$ (95\% CI 78-98\%), a specificity of $82 \%$ (95\% CI 74-88 \%) a positive likelihood ratio of 4.89 (95\% CI 3.29-7.27), a negative likelihood ratio of 0.10 (95\% CI 0.04-0.31), a diagnostic OR of 52.62 (95 \% CI 14.46-191.51), and a receiving operating curve (ROC) of 0.94 (standard error 0.05) [57, 58, 65]. Interestingly, spot sign detected in the arterial phase of CTA is associated with greater absolute hematoma expansion [64]. These results support the use of $\mathrm{CT}$ protocols that include non-contrast $\mathrm{CT}$,

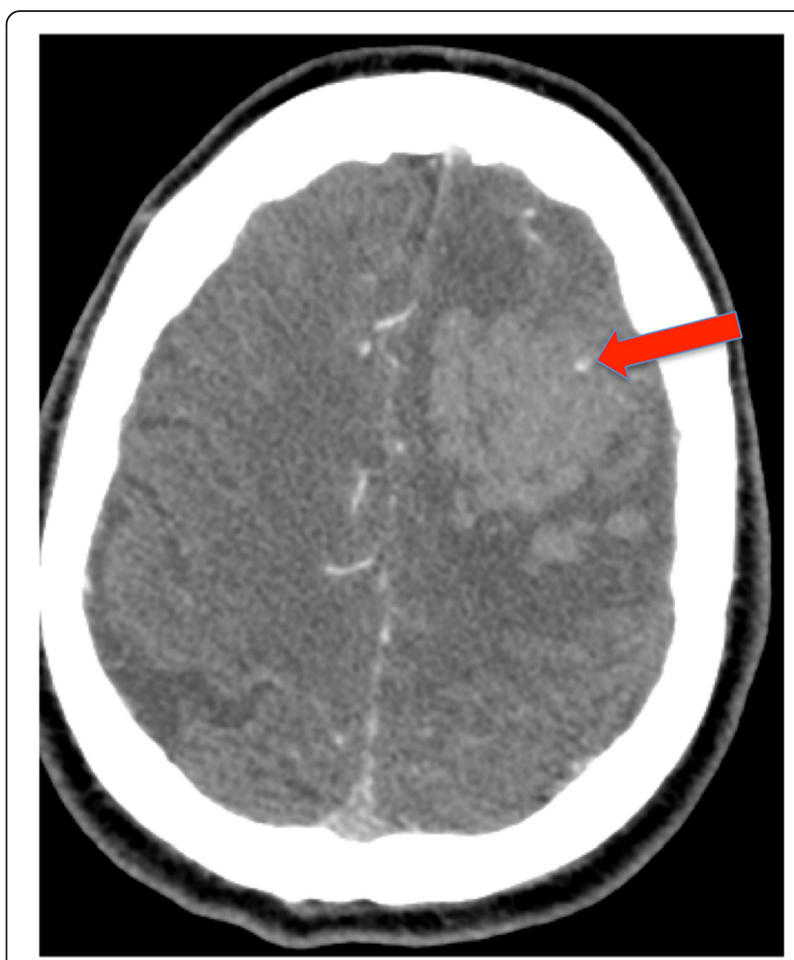

Fig. 3 Spot sign. Initially described as contrast extravasation on CTA the term has evolved to encompass foci of enhancement within the hematoma on CTA (red arrow) 
followed by both CTA and post-contrast study [53]. Identification of a spot sign on CT may have several clinical implications:

A. Identification of contrast extravasation and the spot sign are potent and independent predictors of hematoma expansion [60]. In the multicenter prospective "Prediction of hematoma growth and outcome in patients with ICH using the CTangiography spot sign" (PREDICT) study [66], the presence of spot sign was associated with a relative risk of 2.3 (95 \% CI 1.6-3.1) for hematoma expansion, defined as an absolute increase $>6 \mathrm{ml}$ or a relative increase $>33 \%$ from baseline $\mathrm{ICH}$ volume. However, identification of spot sign does not necessarily infer definite hematoma expansion. A spot sign score has been developed to help predict hematoma expansion [62]. The score includes: number of spot signs (1-2 or $\geq 3)$, maximum axial dimension (1-4 $\mathrm{mm}$ or $\geq 5 \mathrm{~mm}$ ), and maximum attenuation in Hounsfield Units (120-179 HU or $\geq 180 \mathrm{HU})$. A score of 0 indicates that no spot sign was identified on CTA, and it has been associated with minimum risk of hematoma expansion (2\%). In patients with the maximum score (4 points), the risk for hematoma expansion approaches $100 \%$ [62].

B. CTA spot sign is associated with both functional outcome and mortality. In the PREDICT study [66] patients with evidence of a spot sign on CTA had a median 3-month modified Rankin Scale (mRS) of 5 (severe disability-patient bedridden, incontinent, and requiring constant nursing care and attention), in contrast to a median mRS of 3 (moderate disability-patient requiring some help, but able to walk without assistance) in spotsign-negative patients $(p<0.001)$. The 3 -month mortality was $43.4 \%$ in the spot-sign-positive group, compared with $19.6 \%$ in the spot-signnegative patients (HR 2.4, 95 \% CI 1.4-4.0, $p=0.002)$. Likewise, in the spot sign score study [63], the presence of spot sign was associated with an increased risk of in-hospital mortality (55.6\%, OR 4.0, $95 \%$ CI 2.6-5.9, $p<0.0001)$ and with an unfavorable functional outcome at 3 months (50.8 \%, OR 2.5, $95 \%$ CI 1.4-4.3, $p<0.0014$ ), defined as $\mathrm{mRS} \geq 4$ (moderate severe disabilitypatient unable to walk unassisted and unable to attend to own bodily needs without assistance).

C. Patients with positive spot sign on CTA may benefit from more aggressive treatment to reduce hematoma expansion, which may decrease mortality and improve functional outcome [60]. Studies are enrolling spot-sign-positive patients for treatment with recombinant factor VIIa-Spot
Sign for Predicting and Treating ICH Growth (STOP-IT) (ClinicalTrials.gov NCT00810888), and Spot Sign Selection of Intracerebral Hemorrhage to Guide Hemostatic Therapy (SPOTLIGHT) (ClinicalTrials.gov NCT01359202) - and for aggressive antihypertensive treatment (SCOREIT: NIH-NINDS R01NS073344) [67] (Additional file 1: Table S4).

\section{Clinical severity assessment}

Routine use of ICH clinical grading scales to assess baseline severity is useful in the standardization of initial assessment and in communication between providers. Scoring systems may allow for outcome risk stratification based on patients' presentation characteristics (Class I recommendation) [9]. Several clinical grading scales have been developed to risk-stratify and predict outcome after spontaneous ICH [68]. The ICH score (Table 1) [38] is the most widely used and externally validated ICH grading scale [68]. The ICH score was derived from a retrospective analysis of 152 spontaneous $\mathrm{ICH}$ patients who presented to the University of California, San Francisco. Five independent predictors of 30-day mortality were identified and used to build the score: level of consciousness according to the GCS; age; ICH volume; IVH; and

Table 1 Original ICH score and predicted 30-day mortality according to total score

\begin{tabular}{|c|c|c|c|}
\hline Component & Points & Total ICH score & 30-day mortality (\%) \\
\hline \multicolumn{4}{|c|}{ Glasgow Coma Scale } \\
\hline $3-4$ & 2 & 0 & $0-10$ \\
\hline $5-12$ & 1 & & \\
\hline $13-15$ & 0 & & \\
\hline \multicolumn{4}{|l|}{ Age (years) } \\
\hline$\geq 80$ & 1 & 1 & $7-13$ \\
\hline$<80$ & 0 & & \\
\hline \multicolumn{4}{|l|}{$\mathrm{ICH}$ volume (ml) } \\
\hline$\geq 30$ & 1 & 2 & $30-44$ \\
\hline$<30$ & 0 & & \\
\hline \multicolumn{4}{|c|}{ Presence of intraventricular hemorrhage } \\
\hline Yes & 1 & 3 & $56-78$ \\
\hline No & 0 & & \\
\hline \multicolumn{4}{|c|}{ Infra-tentorial origin of $\mathrm{ICH}$} \\
\hline Yes & 1 & 4 & $70-100$ \\
\hline No & 0 & & \\
\hline Total ICH score & $0-6$ & $5-6$ & 100 \\
\hline
\end{tabular}

The five independent predictors of 30-day mortality according to the original $\mathrm{ICH}$ score are displayed in the first column (Glasgow Coma Scale, age, ICH volume, intraventricular hemorrhage, and infra-tentorial location of $\mathrm{ICH}$ ). The total score is the sum of the five components, varying from 0 to 6 points (column 3). The higher the total score (column 3), the higher the predicted 30-day mortality (column 4) $\mathrm{ICH}$ intracerebral hemorrhage 
infra-tentorial location of ICH (Table 1) [38]. The GCS at the time of transfer from the ED to the ICU (or to the operating room) was found to be the strongest independent predictor of 30-day mortality, and consequently given the heaviest weight in the score [38]. The other four components have similar strength of association to outcome, and therefore the same weight in the grading scale. The choice of an ICH volume of $30 \mathrm{ml}$ was based on previously published data [37]; larger volumes $(\geq 60 \mathrm{ml})$ did not improve the score's performance, possibly due to significant confounding effects of other score components (e.g., GCS) on larger hematomas [38]. The higher the score, the higher the mortality, which ranges from $0-10 \%$ in patients with an ICH score of 0 to $100 \%$ in patients with an ICH score $\geq 5$ [68]. However, the use of clinical grading scales such as the ICH score should never be used in isolation to limit interventions in the very acute initial management of patients with $\mathrm{ICH}$.

\section{Patient disposition}

Patients with ICH present management challenges from both a general medical and a neurological perspective, and they are at high risk of early deterioration $[44,45]$. These patients may therefore benefit from initial monitoring and management in dedicated neuroscience ICUs or dedicated stroke units (Class I recommendation) $[9,69,70]$. In the INTERACT 2 study, fewer than $40 \%$ of both study groups were cared for in an ICU, with the majority of patients admitted to a stroke unit rather than an ICU [71]. In a Swedish study including 105,043 patients (all types of acute stroke) from 86 hospitals, care in a stroke unit for patients with $\mathrm{ICH}$ was associated with lower risk of death or institutional living after 3 months (OR 0.60, 95 \% CI 0.54-0.68) compared with other types of wards [72]. ICH patients were among the subgroups with the best relative effect (hazard ratio (HR) for death $0.61,95 \%$ CI 0.58-0.65). A systematic review and metaanalysis of eight clinical trials comparing stroke unit care with general ward care (2657 patients), showed that stroke unit care reduced death or dependency (risk ratio (RR) 0.81, $95 \%$ CI 0.471-0.92, $p=0.0009, I^{2}=60 \%$ ) [70]. Patients requiring advanced monitoring and complex care such as external ventriculostomy drainage, ICP monitoring, mechanical ventilation, or multimodal neuromonitoring often require admission to the ICU.

\section{Medical management of ICH}

Several issues regarding medical and surgical management of ICH remain unanswered. Recent clinical trials examining hemostatic therapy, blood pressure control, and hematoma evacuation have advanced our understanding of ICH management.

\section{Blood pressure management}

Chronic hypertension is the main risk factor for the development of spontaneous $\mathrm{ICH}$, which makes blood pressure (BP) lowering physiologically intuitive as a strategy to prevent hematoma expansion. However, physiologic concern has been that excessive blood pressure reduction could decrease cerebral perfusion pressure (CPP) in the ischemic penumbra. Clinical studies, however, have shown that this concern over the zone of perihematoma ischemia is not well substantiated clinically [73, 74]. Kate et al. [75] randomized ICH patients to two different SBP targets (aggressive group $<150 \mathrm{mmHg}$, and conservative group $<180 \mathrm{mmHg}$ ). Patients underwent CT perfusion study 2 hours after randomization, and the raw imaging data were used to calculate cerebral blood flow (CBF), maximum oxygen extraction fraction $\left(\mathrm{OEF}^{\mathrm{max}}\right)$, and the maximum cerebral metabolic rate of oxygen $\left(\mathrm{CMRO}_{2}\right)$. Despite significant difference in SBP levels between the two groups $(140.5 \pm 18.7 \mathrm{mmHg}$ vs $163.0 \pm 10.6 \mathrm{mmHg}, p<0.001)$, perihematoma CBF, $\mathrm{OEF}^{\max }$, and $\mathrm{CMRO}_{2}$ were not affected by aggressive $\mathrm{BP}$ lowering [75]. Recently published clinical data have further helped clarify the issue of BP thresholds in ICH. In the INTERACT pilot study [76], spontaneous ICH patients within 6 hours of onset were randomly assigned to early intensive BP-lowering strategy (target SBP = $140 \mathrm{mmHg}$ ) or "classic" BP management (target SBP = $180 \mathrm{mmHg}$ ). The study showed that acute aggressive BP lowering was feasible and safe, with a marginal attenuation in hematoma growth $(1.7 \mathrm{ml})$. This pilot study was followed by the INTERACT 2 trial (a phase III trial), which enrolled 2794 patients with spontaneous ICH within 6 hours of hemorrhage [71]. Patients were randomized to the SBP targets (aggressive treatment $<140 \mathrm{mmHg}$ vs standard target $<180 \mathrm{mmHg}$ ). No differences in the primary composite outcome (mortality or major disability) were found ( $52 \%$ vs $55.6 \%, p=0.06$ ). Moreover, early intensive BP reduction was not associated with decreased in hematoma growth, the main mechanism by which aggressive BP treatment was believed to improve outcome. However, a predefined ordinal analysis showed lower mRS scores with intensive treatment (OR for greater disability $0.87,95 \% \mathrm{CI} 0.77-1.00, p=0.04$ ). Two possible conclusions can be drawn from this study: aggressive blood pressure lowering $(\mathrm{SBP}<140 \mathrm{mmHg}$, ideally within 1 hour from presentation) seems to be safe in the acute phase of ICH (Class I; Level of Evidence A); and a lower blood pressure target might have an impact on long-term outcome, even if smaller than anticipated (Class IIa; Level of Evidence B) [9]. The small effect on hematoma growth and composite outcome observed in the INTERACT 2 trial may be explained by small hematoma volumes $(11 \mathrm{ml})$, and, more importantly, by significant delay in BP treatment initiation (median of 4 hours from 
ictus) and delayed achievement in blood pressure target in the treatment group (6 hours of treatment). Therefore, it may be extrapolated that approximately 10 hours passed from hemorrhage onset to target BP achievement. By that time, hematomas would probably have already expanded. Additionally, only $34 \%$ of patients in the treatment group achieved the goal of SBP $<140 \mathrm{mmHg}$ within 1 hour. The Antihypertensive Treatment of Acute Cerebral Hemorrhage (ATACH) 2 trial has been published recently [77], and compared two targets of SBP management in the acute phase of ICH; SBP between 110 and $139 \mathrm{mmHg}$ vs 140 and $179 \mathrm{mmHg}$. The trial was stopped early for futility after the prespecified interim analysis, and included 1000 patients (sample size calculated: 1280 subjects). No difference in the rate of death and disability was found, and the intervention group experienced higher rates of renal adverse events within 7 days $(9.0 \%$ vs $4.0 \%$, $p=0.002)$ [77]. Several differences between INTERACT 2 and ATACH 2 should be mentioned (Table 2). First, the recruitment window was 6 hours in INTERACT 2 vs 3.5 hours (which was extended to 4.5 hours throughout the study) in ATACH 2. The period of blood pressure intervention was 7 days vs 24 hours in the INTERACT 2 and ATACH 2 trials, respectively.

Table 2 INTERACT 2 [71] vs ATACH 2 [77] studies

\begin{tabular}{|c|c|c|c|c|}
\hline & \multicolumn{2}{|c|}{ INTERACT $2(n=2794)$} & \multicolumn{2}{|l|}{ ATACH $2(n=1000)$} \\
\hline & Control & Intervention & Control & Intervention \\
\hline Number of enrolled patients & 1430 & 1399 & 500 & 500 \\
\hline Treatment target (SBP in $\mathrm{mmHg}$ ) & $<180$ & $<140$ & $140-179$ & 110-139 \\
\hline Inclusion criteria & \multicolumn{2}{|l|}{ GCS $>5$} & \multicolumn{2}{|c|}{$\mathrm{ICH}$ (volume $<60 \mathrm{~cm}^{3}$ ), GCS score $\geq 5$} \\
\hline Primary outcome & \multicolumn{2}{|c|}{$\begin{array}{l}\text { Death or major disability (mRS }=3-6 \text { ) at } \\
3 \text { months }\end{array}$} & \multicolumn{2}{|c|}{ Death or disability (mRS $=4-6$ ) at 3 month } \\
\hline Recruitment window & \multicolumn{2}{|l|}{6 hours } & \multicolumn{2}{|l|}{4.5 hours } \\
\hline \multirow[t]{7}{*}{ Medications used to lower blood pressure } & \multicolumn{2}{|l|}{ Urapidil: $32.5 \%$} & \multicolumn{2}{|l|}{ Nicardipine \pm labetalol } \\
\hline & \multicolumn{2}{|c|}{ Nicardipine or nimodipine: $16.2 \%$} & \multirow{2}{*}{\multicolumn{2}{|c|}{$\begin{array}{l}\text { Intravenous diltiazem or urapidil could be } \\
\text { used }\end{array}$}} \\
\hline & \multicolumn{2}{|l|}{ Labetalol: $14.4 \%$} & & \\
\hline & \multicolumn{2}{|l|}{ Nitroglycerin: $14.9 \%$} & & \\
\hline & \multicolumn{2}{|l|}{ Furosemide: $12.4 \%$} & & \\
\hline & \multicolumn{2}{|l|}{ Nitroprusside: $12.1 \%$} & & \\
\hline & \multicolumn{2}{|l|}{ Hydralazine: $5.9 \%$} & & \\
\hline Period of blood pressure intervention & \multicolumn{2}{|l|}{7 days } & \multicolumn{2}{|l|}{24 hours } \\
\hline Time goal of blood pressure lowering & \multicolumn{2}{|l|}{1 hour } & \multicolumn{2}{|l|}{2 hours } \\
\hline Mean interval between symptom onset and randomization & 3.7 hours & 3.7 hours & 3.0 hours & 3.0 hours \\
\hline Systolic blood pressure at presentation $(\mathrm{mmHg})$ & $179 \pm 17$ & $179 \pm 17$ & $201.1 \pm 26.9$ & $200 \pm 27.1$ \\
\hline \multirow[t]{2}{*}{ Mean systolic blood pressure achieved $(\mathrm{mmHg})$} & 164 within 1 hour & 150 within 1 hour & $141.1 \pm 14.8$ ( 2 hours) & $128.9 \pm 16$ (2 hours) \\
\hline & 153 within 6 hours & 139 within 6 hours & & \\
\hline Primary treatment failure ${ }^{a}(\%)$ & \multicolumn{2}{|l|}{66} & \multicolumn{2}{|l|}{12.2} \\
\hline Baseline hematoma volume (ml) & 11 & 11 & 10.2 & 10.3 \\
\hline Asian (\%) & 68.0 & 67.7 & 57.0 & 55.4 \\
\hline Death or disability (\%)-mRS $=3-6$ & 55.6 & 52.0 & 56.1 & 56.2 \\
\hline \multicolumn{5}{|l|}{ Modified Rankin Scale (\%) } \\
\hline 0 & 7.6 & 8.1 & 7.1 & 5.0 \\
\hline 1 & 18.0 & 21.1 & 19.6 & 19.8 \\
\hline 2 & 18.8 & 18.7 & 17.3 & 19.1 \\
\hline 3 & 16.6 & 15.9 & 18.3 & 17.5 \\
\hline 4 & 19.0 & 18.1 & 26.5 & 26.0 \\
\hline 5 & 8.0 & 6.0 & 4.2 & 5.8 \\
\hline 6 & 12.0 & 12.0 & 7.1 & 6.9 \\
\hline
\end{tabular}

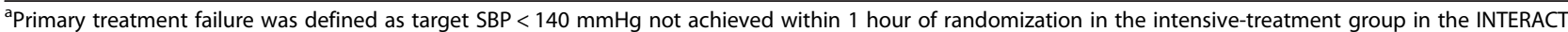
2 trial or within 2 hours in the ATACH 2 trial 
Also, the studies started from different levels of SBP on randomization: $179 \mathrm{mmHg}$ vs $200 \mathrm{mmHg}$ respectively. Importantly, the mean minimum SBP achieved within 2 hours in the control group of ATACH 2 was similar to the mean SBP achieved within 6 hours in the intervention group of INTERACT $2(141 \mathrm{mmHg}$ vs $139 \mathrm{mmHg}$, respectively). In summary: the control group in the ATACH 2 trial achieved similar blood pressure levels compared with the intervention group of INTERACT 2 (141 $\mathrm{mmHg}$ vs $139 \mathrm{mmHg}$ ); the ATACH 2 trial therefore compared $140 \mathrm{mmHg}$ vs $129 \mathrm{mmHg}$; and the patients included in both studies had small hematomas (approximately $10 \mathrm{ml}$ ), making those hematomas less likely to expand [44]. Rapid intensive blood pressure lowering might still be beneficial to patients with higher risk of hematoma expansion (e.g., larger hematomas, positive spot sign) $[44,60]$. In conclusion, acute lowering of SBP to $140 \mathrm{mmHg}$ is safe [9], but it does not seem to improve functional outcome, and SBP levels of $130 \mathrm{mmHg}$ might be associated with increased complications. Additionally, some observational studies have demonstrated the common occurrence of small ischemic lesions on diffusion-weighted MRI after ICH. The impact of these ischemic lesions on functional outcome and their relationship with acute blood pressure lowering vary across those studies, and causality is not well established [78]. Currently, no specific anti-hypertensive agent is considered universally superior. Several agents were used in the INTERACT 2 trial [71], such as an alpha-adrenergic antagonist (urapidil, $32.5 \%$ ), calcium channel blockers (nicardipine or nimodipine, 16.2\%), combined alpha- and beta-blocker (labetalol, 14.4\%), venodilators (nitroglycerin, $14.9 \%$ ), a diuretic (furosemide, $12.4 \%$ ), and arterial vasodilators (nitroprusside, $12.1 \%$; hydralazine $5.9 \%$ ). The ATACH 2 trial administered nicardipine by intravenous infusion, starting at a dose of $5 \mathrm{mg} /$ hour, which was increased by $2.5 \mathrm{mg}$ every 15 minutes (maximum dose of $15 \mathrm{mg}$ /hour), until the target SBP was achieved. Intravenous labetalol was added as second-line agent, if the SBP target was not reached, despite the maximum dose of nicardipine [77]. Nicardipine and labetalol are the most common drugs used in North America; both agents appear to be safe [79], but nicardipine might be more effective in reaching and maintaining the target BP [80]. However, local drug availability and drug approval is a practical consideration.

\section{Anticoagulant-associated ICH}

The use of anticoagulants has significantly increased in the last decades, leading to a 3-fold increase in the incidence of anticoagulant-related intracranial hemorrhage [14]. Patients experiencing $\mathrm{ICH}$ on antithrombotic agents have an increased risk for hematoma expansion and higher risk of death and poor outcome [24, 81]. In
AAICH, the main goals are: withholding the culprit drug; reversing the drug effect by the administration of antidote when available; and monitoring the effectiveness of anticoagulation reversal with laboratory tests. However, the laboratory correction of coagulopathy may not be associated with the reversal of coagulopathy in vivo [40].

a. Warfarin is responsible for 9-14\% of all cases of $\mathrm{ICH}$ [82], with an annual risk of warfarin-related ICH of between 0.3 and $3.7 \%$ when the INR is between 2 and 4.5 [83] (Table 3). Patients on long-term warfarin have up to an 11-fold higher risk of $\mathrm{ICH}$ compared with patients not taking anticoagulants [84]. Increased age ( $\geq 70$ years) [84, 85], a history of chronic hypertension [85], and the concomitant use of antiplatelet therapy (APT) [86] are risk factors for warfarin-related $\mathrm{ICH}$. Bleeding risk scores (e.g., HAS-BLED) may help assess the risk of major bleeding in patients with atrial fibrillation [87]. Warfarin-related $\mathrm{ICH}$ is also associated with larger hematoma size [88] and higher mortality rates compared with patients without coagulopathy [81, 89]. For patients with warfarin-related ICH and elevated INR $(>1.4)$, urgent coagulopathy reversal is warranted. Vitamin $\mathrm{K}$, given as a slow intravenous infusion (5-10 $\mathrm{mg}$ over 30 minutes), can completely reverse the warfarin effect. However, it can take up to 24 hours to completely reverse warfarin effect, and so its use as sole therapy is associated with increased risk of hematoma expansion, and is not recommended [9]. Currently, there are two other treatment options to acutely reverse the warfarin effect: the transfusion of fresh frozen plasma (FFP) or the use of prothrombin complex concentrate (PCC). FFP contains all coagulation factors but several transfusion-related complications, such as allergic reactions, possibility of infectious disease transmission, transfusion-related acute lung injury and transfusion-related circulatory overload could occur. More importantly, the time consumed to thaw, cross-match, and transfuse the appropriate dose $(10-40 \mathrm{ml} / \mathrm{kg})$ makes the use of FFP for the acute reversal of warfarin-related $\mathrm{ICH}$ less appealing and less effective than the use of PCC [90]. PCC is a virally inactivated preparation of concentrated coagulation factors pooled from healthy donors. Some PCC preparations contain factors II, IX and X (three-factor PCC), while some other preparations also contain higher concentrations of factor VII (four-factor PCC). Coagulation factors can be presented in the inactivated or activated forms, depending on the formulation (Additional file 1). Dosing is usually based on factor IX 
Table 3 Anticoagulants and reversal strategies

\begin{tabular}{|c|c|c|c|c|c|c|c|}
\hline Drug & Target & $\begin{array}{l}\text { Elimination and } \\
\text { half-life (hours) }\end{array}$ & Rate of $\mathrm{ICH}$ & $\begin{array}{l}\text { Monitoring } \\
\text { coagulation tests }\end{array}$ & Antidote and reversal & $\begin{array}{l}\text { Possible } \\
\text { intervention }\end{array}$ & Guidelines \\
\hline \multicolumn{8}{|c|}{ Vitamin $\mathrm{K}$ antagonist } \\
\hline \multirow[t]{3}{*}{ Warfarin } & \multirow[t]{3}{*}{$\begin{array}{l}\text { Factors II, VIII, IX, X; } \\
\text { proteins } C, S\end{array}$} & $\begin{array}{l}\text { Hepatic } \\
\text { metabolism }\end{array}$ & \multirow[t]{3}{*}{$0.3-1.1 \%[90]$} & \multirow[t]{3}{*}{$\begin{array}{l}\text { Good linear } \\
\text { correlation PT/INR }\end{array}$} & Vitamin $\mathrm{K}$ & \multirow[t]{3}{*}{ Not dialyzable } & $\begin{array}{l}\text { Withhold VKA + intravenous vitamin K+ } \\
\text { replace vitamin K-dependent factors (three- } \\
\text { or four-factor PCC IV or FFP if PCCS are not } \\
\text { available), and correct the INR (keep INR }< \\
\text { 1.4) (Class I; Level of Evidence C) [9] }\end{array}$ \\
\hline & & $\begin{array}{l}92 \% \text { renal } \\
\text { elimination }\end{array}$ & & & $\begin{array}{l}\text { Vitamin } \mathrm{K} 10 \mathrm{mg} \text { IV } \\
\text { associated with 4-FPCC } \\
20 \mathrm{IU} / \mathrm{kg} \text { (or FFP = 10- } \\
15 \mathrm{ml} / \mathrm{kg} \text {, if PCC is not } \\
\text { available) }\end{array}$ & & $\begin{array}{l}\text { PCCs might be considered over FFP } \\
\text { (Class Ilb; Level of Evidence B) [9] }\end{array}$ \\
\hline & & $20-60$ & & & Goal: INR < 1.4 [90] & & $\begin{array}{l}\text { rFVIla is not recommended for VKA reversal } \\
\text { in ICH (Class III; Level of Evidence C) [9] }\end{array}$ \\
\hline \multicolumn{8}{|c|}{ Unfractionated heparin, LMWHs, and heparinoids } \\
\hline \multirow[t]{3}{*}{ UFH } & \multirow{3}{*}{$\begin{array}{l}\text { Binds and activates } \\
\text { antithrombin (which } \\
\text { blocks coagulation } \\
\text { factors Xa and lla). By } \\
\text { inactivating thrombin, } \\
\text { heparin prevents fibrin } \\
\text { formation }\end{array}$} & Renal & \multirow[t]{3}{*}{$\begin{array}{l}0.1 \text { to } 0.2 \% \\
{[90,151]}\end{array}$} & \multirow[t]{3}{*}{$\begin{array}{l}\text { Good linear } \\
\text { correlation aPTT }\end{array}$} & \multirow[t]{3}{*}{$\begin{array}{l}\text { Protamine sulfate } 1 \mathrm{mg} \text { of } \\
\text { protamine per } 100 \text { units of } \\
\text { UFH infused over the } \\
\text { preceding } 3 \text { hours }\end{array}$} & \multirow[t]{3}{*}{ Not dialyzable } & $\begin{array}{l}\text { Protamine sulfate }-1 \mathrm{mg} \text { for every } 100 \text { units } \\
\text { of heparin given in the previous } 2-3 \text { hours } \\
\text { with a maximum single dose of } 50 \mathrm{mg} \\
\text { (Strong recommendation, moderate quality } \\
\text { evidence) [90] }\end{array}$ \\
\hline & & $\begin{array}{l}0.5-2.5 \text { (dose } \\
\text { dependent) }\end{array}$ & & & & & $\begin{array}{l}\text { If aPTT is still elevated, repeat administration } \\
\text { of protamine at a dose of } 0.5 \mathrm{mg} \text { protamine } \\
\text { per } 100 \text { units of UFH (Conditional } \\
\text { recommendation, low quality of evidence) } \\
\text { [90] }\end{array}$ \\
\hline & & & & & & & $\begin{array}{l}\text { Reversal of prophylactic SC heparin only if } \\
\text { aPTT is significantly prolonged (Good } \\
\text { Practice statement) [90] }\end{array}$ \\
\hline \multirow[t]{4}{*}{ Enoxaparin } & \multirow{4}{*}{$\begin{array}{l}\text { LMWH } \\
\text { Binds and activates } \\
\text { antithrombin (which } \\
\text { blocks coagulation } \\
\text { factors Xa and Ila) }\end{array}$} & $40 \%$ renal & \multirow{4}{*}{$\begin{array}{l}0.2-0.5 \% \text { [98] } \\
\text { Enoxaparin } 1 \mathrm{mg} / \\
\text { kg BID; bridging } \\
\text { warfarin with } \\
\text { target INR 2-3 }\end{array}$} & \multirow[t]{4}{*}{ Anti-factor Xa } & \multirow{4}{*}{$\begin{array}{l}\text { Protamine sulfate partially } \\
\text { reverses }(60 \%) \text { LMWH } \\
\text { effect. One mg protamine } \\
\text { for every } 1 \mathrm{mg} \text { enoxaparin }\end{array}$} & \multirow[t]{4}{*}{ Not dialyzable } & $\begin{array}{l}\text { Strong recommendation, moderate quality } \\
\text { evidence [90] }\end{array}$ \\
\hline & & 4.5 hours & & & & & $\begin{array}{l}\text { Protamine sulfate } 1 \mathrm{mg} \text { per } 1 \mathrm{mg} \text { of } \\
\text { enoxaparin (maximum single dose of } \\
50 \mathrm{mg} \text {-if enoxaparin was given within } \\
8 \text { hours) }\end{array}$ \\
\hline & & & & & & & $\begin{array}{l}\text { Protamine sulfate } 0.5 \mathrm{mg} \text { of protamine per } \\
1 \mathrm{mg} \text { of enoxaparin (if enoxaparin was } \\
\text { given within } 8-12 \text { hours) }\end{array}$ \\
\hline & & & & & & & After 12 hours, protamine is not needed \\
\hline \multirow[t]{3}{*}{ Dalteparin } & \multirow{3}{*}{$\begin{array}{l}\text { LMWH } \\
\text { Binds and activates } \\
\text { antithrombin (which } \\
\text { blocks coagulation } \\
\text { factors Xa and Ila) }\end{array}$} & Renal & \multirow[t]{3}{*}{ Not established } & \multirow[t]{3}{*}{ Anti-factor Xa } & \multirow{3}{*}{$\begin{array}{l}\text { Protamine sulfate partially } \\
\text { reverses }(60 \%) \text { LMWH } \\
\text { effect. One mg protamine } \\
\text { for every } 100 \text { anti-Xa IU } \\
\text { dalteparin }\end{array}$} & \multirow[t]{3}{*}{ Not dialyzable } & \multirow{3}{*}{$\begin{array}{l}\text { Protamine sulfate } 1 \mathrm{mg} \text { per } 100 \mathrm{IU} \text { of } \\
\text { dalteparin administered in the past 3-5 } \\
\text { half-lives (maximum } 50 \mathrm{mg} \text { ) (Strong } \\
\text { recommendation, moderate quality } \\
\text { evidence) [90] }\end{array}$} \\
\hline & & 2.5 hours & & & & & \\
\hline & & $\begin{array}{l}3.7-7.7 \text { hours with } \\
\text { RF }\end{array}$ & & & & & \\
\hline
\end{tabular}

Unfractionated heparin, LMWHs, and heparinoids mbin (which blocks coagulation dalteparin 
Table 3 Anticoagulants and reversal strategies (Continued)

\begin{tabular}{|c|c|c|c|c|c|c|c|}
\hline Nadroparin & $\begin{array}{l}\text { LMWH } \\
\text { Binds and activates } \\
\text { antithrombin (which } \\
\text { blocks coagulation } \\
\text { factors Xa and lla) }\end{array}$ & $\begin{array}{l}\text { Renal } \\
3.5 \text { hours }\end{array}$ & Not established & Anti-factor Xa & $\begin{array}{l}\text { Protamine sulfate partially } \\
\text { reverses }(60 \%) \text { LMWH } \\
\text { effect. One } \mathrm{mg} \text { protamine } \\
\text { for every } 100 \text { anti-Xa IU } \\
\text { nadroparin }\end{array}$ & Not dialyzable & $\begin{array}{l}\text { Protamine sulfate } 1 \mathrm{mg} \text { per } 100 \mathrm{IU} \text { of } \\
\text { nadroparin administered in the past } 3-5 \\
\text { half-lives (maximum } 50 \mathrm{mg} \text { ) (Strong recom- } \\
\text { mendation, moderate quality evidence) [90] }\end{array}$ \\
\hline \multicolumn{8}{|l|}{ Pentasaccharides } \\
\hline \multirow[t]{3}{*}{$\begin{array}{l}\text { Fondaparinux } \\
\text { (Aristra })^{\oplus}\end{array}$} & \multirow{3}{*}{$\begin{array}{l}\text { Binds with } \\
\text { antithrombin and } \\
\text { potentiates inhibition } \\
\text { of free factor Xa, } \\
\text { preventing formation } \\
\text { of the prothrombinase } \\
\text { complex }\end{array}$} & 50-77\% renal & \multirow[t]{3}{*}{ Not established } & \multirow[t]{3}{*}{ Anti-factor Xa } & \multirow[t]{3}{*}{ None } & $\begin{array}{l}\text { Activated PCC } \\
\text { (FEIBA } 20 \text { units/ } \\
\text { kg) }\end{array}$ & $\begin{array}{l}\text { aPCC } 20 \text { IU/kg (Conditional } \\
\text { recommendation, low-quality evidence) [90] }\end{array}$ \\
\hline & & $17-21$ hours & & & & \multirow[t]{2}{*}{$\begin{array}{l}\text { Dialyzable } \\
\text { (clearance } \\
\text { increased by } \\
20 \% \text { ) }\end{array}$} & $\begin{array}{l}\text { rFVIla }(90 \mu \mathrm{g} / \mathrm{kg}) \text { if aPCC is not available } \\
\text { (Conditional recommendation, low-quality } \\
\text { evidence) [90] }\end{array}$ \\
\hline & & $\begin{array}{l}\text { Prolonged in } \\
\text { older patients and } \\
\text { in RF }\end{array}$ & & & & & $\begin{array}{l}\text { Protamine sulfate is not recommended } \\
\text { (Strong recommendation, low-quality } \\
\text { evidence) [90] }\end{array}$ \\
\hline \multicolumn{8}{|c|}{ Direct thrombin (factor lla) inhibitors } \\
\hline $\begin{array}{l}\text { Argatroban } \\
\left(\text { Acova }^{\oplus}\right)\end{array}$ & $\begin{array}{l}\text { Competitive direct } \\
\text { inhibition of thrombin } \\
\text { (factor lla) including } \\
\text { thrombin-mediated } \\
\text { platelet activation and } \\
\text { aggregation }\end{array}$ & $\begin{array}{l}\text { No renal excretion } \\
0.75 \text { hours } \\
\text { (prolonged in } \\
\text { hepatic } \\
\text { dysfunction) }\end{array}$ & Not established & aPTT, ACT & None & $\begin{array}{l}\text { Activated PCC } \\
\text { (FEIBA 50-80 } \\
\text { units/kg) } \\
\text { Antifibrinolytic } \\
\text { agent (e.g., } \\
\text { tranexamic acid, } \\
\text { epsilon- } \\
\text { aminocaproic } \\
\text { acid) } \\
\text { Hemodialysis } \\
\text { (approximately } \\
20 \% \text { over } \\
4 \text { hours) }\end{array}$ & $\begin{array}{l}\text { aPCC (50 units/kg) or four-factor PCC } \\
\text { ( } 50 \text { units } / \mathrm{kg} \text { ) (Conditional recommendation, } \\
\text { low-quality evidence) [90] } \\
\text { rFVlla or FFP are not recommended in direct } \\
\text { thrombin inhibitor-related ICH (Strong } \\
\text { recommendation, low-quality evidence) [90] }\end{array}$ \\
\hline \multirow[t]{4}{*}{ Bivalirudin } & \multirow{4}{*}{$\begin{array}{l}\text { Reversible direct } \\
\text { inhibition of thrombin } \\
\text { (factor lla) including } \\
\text { thrombin-mediated } \\
\text { platelet activation and } \\
\text { aggregation }\end{array}$} & $20 \%$ renal & \multirow[t]{4}{*}{$0.1 \%[151]$} & \multirow[t]{4}{*}{$\begin{array}{l}\mathrm{ECT} \text { (PT, aPTT, ACT } \\
\text { has nonlinear } \\
\text { prolongation) }\end{array}$} & \multirow[t]{4}{*}{ None } & $\begin{array}{l}\text { Activated PCC } \\
\text { (FEIBA 50-80 } \\
\text { units } / \mathrm{kg} \text { ) }\end{array}$ & \\
\hline & & $\begin{array}{l}0.5 \text { (prolonged in } \\
\text { renal impairment) }\end{array}$ & & & & $\begin{array}{l}\text { Antifibrinolytic } \\
\text { agent (e.g., } \\
\text { tranexamic acid, } \\
\text { epsilon- } \\
\text { aminocaproic } \\
\text { acid) }\end{array}$ & $\begin{array}{l}\text { aPCC ( } 50 \text { units } / \mathrm{kg}) \text { or four-factor PCC } \\
(50 \text { units } / \mathrm{kg} \text { ) (Conditional recommendation, } \\
\text { low-quality evidence) }\end{array}$ \\
\hline & & $\begin{array}{l}\text { GFR } 30-59, \\
34 \text { minutes }\end{array}$ & & & & \multirow{2}{*}{$\begin{array}{l}\text { Hemodialysis } \\
\text { (approximately } \\
25 \% \text { over } \\
4 \text { hours) }\end{array}$} & \multirow{2}{*}{$\begin{array}{l}\text { rFVIla or FFP are not recommended in direct } \\
\text { thrombin inhibitor-related ICH (Strong } \\
\text { recommendation, low-quality evidence) }\end{array}$} \\
\hline & & $\begin{array}{l}\text { GFR 10-29, } \\
57 \text { minutes }\end{array}$ & & & & & \\
\hline $\begin{array}{l}\text { Dabigatran } \\
\left(\text { Pradaxa }^{\oplus}\right)\end{array}$ & $\begin{array}{l}\text { Reversible direct } \\
\text { inhibition of thrombin } \\
\text { (factor Ila) including }\end{array}$ & $>80 \%$ renal & $\begin{array}{l}0.30 \%(150 \mathrm{mg}) \\
{[98]}\end{array}$ & $\begin{array}{l}\text { Modified TT/ECT/ } \\
\text { prolongs PT } \\
\text { linearly with }\end{array}$ & $\begin{array}{l}\text { Idarucizumab or Praxbind } \\
\text { (humanized antibody } \\
\text { fragment against }\end{array}$ & $\begin{array}{l}\text { Activated PCC } \\
\text { (FEIBA 50-80 } \\
\text { units } / \mathrm{kg} \text { ) }\end{array}$ & $\begin{array}{l}\text { Idarucizumab } 5 \mathrm{~g} \text { IV in two divided doses if } \\
\text { dabigatran was administered within 3-5 } \\
\text { half-lives and no RF (Strong }\end{array}$ \\
\hline
\end{tabular}


Table 3 Anticoagulants and reversal strategies (Continued)

\begin{tabular}{|c|c|c|c|c|c|c|c|}
\hline & \multirow[t]{6}{*}{$\begin{array}{l}\text { thrombin-mediated } \\
\text { platelet activation and } \\
\text { aggregation }\end{array}$} & & & $\begin{array}{l}\text { increasing serum } \\
\text { levels, while aPTT } \\
\text { is affected in a } \\
\text { nonlinear way }\end{array}$ & $\begin{array}{l}\text { dabigatran), in two doses } \\
\text { of } 2.5 \mathrm{~g} \mathrm{IV} 15 \text { minutes } \\
\text { apart }\end{array}$ & & $\begin{array}{l}\text { recommendation, moderate quality of } \\
\text { evidence) or in the presence of RF leading } \\
\text { to continued drug exposure beyond the } \\
\text { normal } 3-5 \text { half-lives (Strong recommenda- } \\
\text { tion, moderate quality of evidence) }\end{array}$ \\
\hline & & $12-17$ hours & $\begin{array}{l}0.23 \%(110 \mathrm{mg}) \\
{[98]}\end{array}$ & & & \multirow{5}{*}{$\begin{array}{l}\text { Activated } \\
\text { charcoal if last } \\
\text { dose was taken } \\
<2 \text { hours } \\
\text { Hemodialysis } \\
\text { (approximately } \\
57 \% \text { over } \\
4 \text { hours) } \\
\text { Antifibrinolytic } \\
\text { agent (e.g.r } \\
\text { tranexamic acid, } \\
\text { epsilon- } \\
\text { aminocaproic } \\
\text { acid) }\end{array}$} & \multirow{5}{*}{$\begin{array}{l}\text { Hemodialysis if idarucizumab is not available } \\
\text { (Conditional recommendation, low-quality } \\
\text { data) }\end{array}$} \\
\hline & & $\begin{array}{l}16.6 \text { hours in mild } \\
\text { RF }\end{array}$ & \multirow{4}{*}{$\begin{array}{l}\text { ICH distribution: } \\
46 \% \\
\text { intraparenchymal, } \\
45 \% \mathrm{SDH} \text {, and } \\
8 \% \mathrm{SAH}[90]\end{array}$} & & & & \\
\hline & & $\begin{array}{l}18.7 \text { hours in } \\
\text { moderate RF }\end{array}$ & & & & & \\
\hline & & $\begin{array}{l}27.5 \text { hours in } \\
\text { severe RF }\end{array}$ & & & & & \\
\hline & & $\begin{array}{l}34.1 \text { hours in } \\
\text { patients on } \\
\text { hemodialysis }\end{array}$ & & & & & \\
\hline Desirudin & $\begin{array}{l}\text { Irreversible direct } \\
\text { inhibition of thrombin } \\
\text { (factor Ila) including } \\
\text { thrombin-mediated } \\
\text { platelet activation and } \\
\text { aggregation }\end{array}$ & $\begin{array}{l}40-50 \% \text { renal } \\
2 \text { hours ( } 12 \text { hours } \\
\text { with renal } \\
\text { impairment) }\end{array}$ & Not established & aPTT & None & Dialyzable & $\begin{array}{l}\text { aPCC (50 units/kg) or four-factor PCC } \\
(50 \text { units/kg) (Conditional recommendation, } \\
\text { low-quality evidence) [90] } \\
\text { rFVlla or FFP are not recommended in direct } \\
\text { thrombin inhibitor-related ICH (Strong } \\
\text { recommendation, low-quality evidence) [90] }\end{array}$ \\
\hline \multicolumn{8}{|c|}{ Direct factor Xa inhibitors } \\
\hline $\begin{array}{l}\text { Apixaban } \\
\text { (Eliquis }^{\oplus} \text { ) }\end{array}$ & $\begin{array}{l}\text { Prevents factor Xa- } \\
\text { mediated conversion of } \\
\text { prothrombin to } \\
\text { thrombin }\end{array}$ & $\begin{array}{l}\text { Mainly fecal } \\
27 \% \text { renal } \\
8-14 \text { hours }\end{array}$ & $\begin{array}{l}\text { Apixaban } 5 \mathrm{mg} \\
\text { twice daily } \\
0.33 \% / \text { year [98] }\end{array}$ & $\begin{array}{l}\text { Anti-factor Xa } \\
\text { There are scant } \\
\text { data regarding } \\
\text { the effect of } \\
\text { apixaban on } \\
\text { traditional } \\
\text { coagulation tests }\end{array}$ & $\begin{array}{l}\text { Currently, there is no FDA- } \\
\text { approved specific antidote } \\
\text { for this class of } \\
\text { anticoagulants } \\
\text { Antidotes under } \\
\text { investigation: } \\
\text { - Aripazine } \\
\text { (PER977-synthetic small } \\
\text { molecule) } \\
\text { - Andexanet } \\
\text { (PRT064445_recombinant } \\
\text { modified factor Xa protein) }\end{array}$ & $\begin{array}{l}\text { Unactivated } \\
\text { four-factor PCC } \\
\text { (50 units/kg). If } \\
\text { not available, a } \\
\text { three-factor PCC } \\
\text { can be used. } \\
\text { Activated } \\
\text { charcoal if last } \\
\text { dose was taken } \\
<2 \text { hours } \\
\text { Antifibrinolytic } \\
\text { agent (e.g., } \\
\text { tranexamic acid, } \\
\text { epsilon- } \\
\text { aminocaproic } \\
\text { acid) } \\
\text { Minimal removal } \\
\text { with dialysis } \\
\text { (decreased by } \\
14 \% \text { over } \\
4 \text { hours) }\end{array}$ & $\begin{array}{l}\text { Activated charcoal }(50 \mathrm{~g}) \text { within } 2 \text { hours of } \\
\text { ingestion (Conditional recommendation, } \\
\text { very low-quality evidence) [90] } \\
\text { aPCC ( } 50 \text { units/kg) or Four-factor PCC } \\
(50 \mathrm{U} / \mathrm{kg}) \text { or activated PCC }(50 \mathrm{U} / \mathrm{kg}) \text { if ICH } \\
\text { happened within } 3-5 \text { half-lives of drug or if } \\
\text { liver failure (Conditional recommendation, } \\
\text { low-quality evidence) [90] } \\
\text { Four-factor PCC or activated PCC over rFVIla } \\
\text { (Conditional recommendation, low-quality } \\
\text { evidence) [90] }\end{array}$ \\
\hline $\begin{array}{l}\text { Rivaroxaban } \\
\left.\text { (Xarelto }^{\oplus}\right)\end{array}$ & $\begin{array}{l}\text { Prevents factor Xa- } \\
\text { mediated conversion of }\end{array}$ & $\begin{array}{l}66 \% \text { renal } \\
28 \% \text { fecal }\end{array}$ & $\begin{array}{l}\text { Rivaroxaban } \\
20 \text { mg daily }\end{array}$ & Anti-factor Xa & $\begin{array}{l}\text { Currently, there is no FDA- } \\
\text { approved specific antidote }\end{array}$ & $\begin{array}{l}\text { Unactivated } \\
\text { four-factor PCC }\end{array}$ & \\
\hline
\end{tabular}


Table 3 Anticoagulants and reversal strategies (Continued)

\begin{tabular}{|c|c|c|c|c|c|c|c|}
\hline & $\begin{array}{l}\text { prothrombin to } \\
\text { thrombin }\end{array}$ & 7 to 11 hours & $-<0.5 \%$ /year & $\begin{array}{l}\text { Rivaroxaban levels } \\
\text { linearly increase } \\
\text { PT and aPTT levels }\end{array}$ & $\begin{array}{l}\text { for this class of } \\
\text { anticoagulants } \\
\text { Antidotes under } \\
\text { investigation: } \\
\text { - Aripazine } \\
\text { (PER977—synthetic small } \\
\text { molecule) } \\
\text { - Andexanet } \\
\text { (PRT064445_recombinant } \\
\text { modified factor Xa protein) }\end{array}$ & $\begin{array}{l}\text { ( } 50 \text { units/kg). If } \\
\text { not available, a } \\
\text { three-factor PCC } \\
\text { can be used. } \\
\text { Activated } \\
\text { charcoal if last } \\
\text { dose was taken } \\
<2 \text { hours } \\
\text { Antifibrinolytic } \\
\text { agent (e.g., } \\
\text { tranexamic acid, } \\
\text { epsilon- } \\
\text { aminocaproic } \\
\text { acid) } \\
\text { Not dialyzable } \\
\text { (rivaroxaban is } \\
\text { highly protein } \\
\text { bound) }\end{array}$ & $\begin{array}{l}\text { Activated charcoal }(50 \mathrm{~g}) \text { within } 2 \text { hours of } \\
\text { ingestion (Conditional recommendation, } \\
\text { very low-quality evidence) [90] } \\
\text { Four-factor PCC (50 U/kg) or activated PCC } \\
\text { (50 U/kg) if ICH happened within 3-5 half- } \\
\text { lives of drug or if liver failure (Conditional } \\
\text { recommendation, low-quality evidence) [90] } \\
\text { Four-factor PCC or activated PCC over rFVVIla } \\
\text { (Conditional recommendation, low-quality } \\
\text { evidence) [90] }\end{array}$ \\
\hline Edoxaban & $\begin{array}{l}\text { Prevents factor Xa- } \\
\text { mediated conversion of } \\
\text { prothrombin to } \\
\text { thrombin }\end{array}$ & $\begin{array}{l}50 \% \text { renal } \\
10-14 \text { hours }\end{array}$ & $\begin{array}{l}\text { Edoxaban } 60 \mathrm{mg} \\
\text { daily compared } \\
\text { with warfarin (HR } \\
0.54,95 \% \mathrm{Cl} \\
0.38-0.77)[90]\end{array}$ & $\begin{array}{l}\text { There are scant } \\
\text { data regarding } \\
\text { the effect of } \\
\text { edoxaban on } \\
\text { traditional } \\
\text { coagulation tests }\end{array}$ & $\begin{array}{l}\text { Currently, there is no FDA- } \\
\text { approved specific antidote } \\
\text { for this class of } \\
\text { anticoagulants } \\
\text { Antidotes under } \\
\text { investigation: } \\
\text { - Aripazine } \\
\text { (PER977-synthetic small } \\
\text { molecule) } \\
\text { - Andexanet } \\
\text { (PRT064445_recombinant } \\
\text { modified factor Xa protein) }\end{array}$ & $\begin{array}{l}\text { Unactivated } \\
\text { four-factor PCC } \\
\text { ( } 50 \text { units/kg). If } \\
\text { not available, a } \\
\text { three-factor PCC } \\
\text { can be used. } \\
\text { Activated } \\
\text { charcoal if last } \\
\text { dose was taken } \\
<2 \text { hours } \\
\text { Antifibrinolytic } \\
\text { agent (e.g., } \\
\text { tranexamic acid, } \\
\text { epsilon- } \\
\text { aminocaproic } \\
\text { acid) } \\
\text { Not dialyzable } \\
\text { (edoxaban is } \\
\text { highly protein } \\
\text { bound) }\end{array}$ & $\begin{array}{l}\text { Activated charcoal }(50 \mathrm{~g}) \text { within } 2 \text { hours of } \\
\text { ingestion (Conditional recommendation, } \\
\text { very low-quality evidence) [90] } \\
\text { Four-factor PCC (50 U/kg) or activated PCC } \\
\text { (50 U/kg) if ICH happened within 3-5 half- } \\
\text { lives of drug or if liver failure (Conditional } \\
\text { recommendation, low-quality evidence) [90] } \\
\text { Four-factor PCC or activated PCC over rFVVIla } \\
\text { (Conditional recommendation, low-quality } \\
\text { evidence) [90] }\end{array}$ \\
\hline \multicolumn{8}{|l|}{ Antiplatelets } \\
\hline Aspirin & $\begin{array}{l}\text { Irreversible COX-1 and } \\
2 \text { enzyme inhibitor (in- } \\
\text { hibits thromboxane A2) }\end{array}$ & $\begin{array}{l}5.6-35.6 \% \text { renal } \\
0.3 \text { hours }\end{array}$ & $\begin{array}{l}\text { It is unclear if } \\
\text { antiplatelet therapy } \\
\text { increases the } \\
\text { incidence of } \mathrm{ICH}\end{array}$ & $\begin{array}{l}\text { Light Transmission } \\
\text { Platelet } \\
\text { Aggregation with } \\
\text { or without } \\
\text { Secretion }\end{array}$ & $\begin{array}{l}\text { None } \\
\text { The usefulness of platelet } \\
\text { transfusions in ICH patients } \\
\text { with a history of } \\
\text { antiplatelet use is } \\
\text { uncertain }\end{array}$ & $\begin{array}{l}\text { Dialyzable } \\
\text { DDAVP } 0.4 \mu \mathrm{g} / \\
\mathrm{kg}\end{array}$ & $\begin{array}{l}\text { DDAVP } 0.4 \mu \mathrm{g} / \mathrm{kg} \text { IV (Conditional } \\
\text { recommendation, low-quality evidence) [90] } \\
\text { Platelet transfusion is not recommended } \\
\text { (Conditional recommendation, low-quality } \\
\text { evidence) [90] } \\
\text { Platelet transfusion for patients who will } \\
\text { undergo a neurosurgical procedure } \\
\text { (Conditional recommendation, moderate } \\
\text { quality of evidence) [90] } \\
\text { DDAVP can be used in addition to platelet } \\
\text { transfusion in patients who will undergo }\end{array}$ \\
\hline
\end{tabular}
mediated conversion of 10-14 hours prothrombin to

Irreversible COX-1 and $\quad 5.6-35.6 \%$ renal
2 enzyme inhibitor (in- $\quad 0.3$ hours hibits thromboxane A2)
Is inclear if

increases the

increases the transfusions in $\mathrm{ICH}$ patients

with a history of

transfusion in patients who will undergo 
Table 3 Anticoagulants and reversal strategies (Continued)

\begin{tabular}{|c|c|c|c|c|c|c|c|}
\hline Clopidogrel & $\begin{array}{l}\text { Irreversible inhibition of } \\
\text { P2Y12 ADP receptor }\end{array}$ & $\begin{array}{l}50 \% \text { renal } \\
46 \% \text { fecal } \\
6-8 \text { hours }\end{array}$ & $\begin{array}{l}\text { It is unclear if anti } \\
\text { platelet therapy } \\
\text { increases the } \\
\text { incidence of ICH }\end{array}$ & $\begin{array}{l}\text { Light Transmission } \\
\text { Platelet } \\
\text { Aggregation with } \\
\text { or without } \\
\text { Secretion }\end{array}$ & $\begin{array}{l}\text { None } \\
\text { The usefulness of platelet } \\
\text { transfusions in ICH patients } \\
\text { with a history of } \\
\text { antiplatelet use is } \\
\text { uncertain }\end{array}$ & $\begin{array}{l}\text { Not dialyzable } \\
\text { DDAVP } 0.4 \mu \mathrm{g} / \\
\mathrm{kg}\end{array}$ & $\begin{array}{l}\text { DDAVP } 0.4 \mu \mathrm{g} / \mathrm{kg} \text { IV (Conditional } \\
\text { recommendation, low-quality evidence) [90] } \\
\text { Platelet transfusion is not recommended } \\
\text { (Conditional recommendation, low-quality } \\
\text { evidence) [90] } \\
\text { Platelet transfusion for patients who will } \\
\text { undergo a neurosurgical procedure } \\
\text { (Conditional recommendation, moderate } \\
\text { quality of evidence) [90] } \\
\text { DDAVP can be used in addition to platelet } \\
\text { transfusion in patients who will undergo } \\
\text { neurosurgical procedure (Conditional } \\
\text { recommendation, low-quality evidence) [90] }\end{array}$ \\
\hline Prasugrel & $\begin{array}{l}\text { Irreversible inhibition of } \\
\text { P2Y12 ADP receptor }\end{array}$ & $\begin{array}{l}68 \% \text { renal } \\
27 \% \text { fecal } \\
2-15 \text { hours }\end{array}$ & $\begin{array}{l}\text { It is unclear if anti } \\
\text { platelet therapy } \\
\text { increases the } \\
\text { incidence of ICH }\end{array}$ & $\begin{array}{l}\text { Light Transmission } \\
\text { Platelet } \\
\text { Aggregation with } \\
\text { or without } \\
\text { Secretion }\end{array}$ & $\begin{array}{l}\text { None } \\
\text { The usefulness of platelet } \\
\text { transfusions in ICH patients } \\
\text { with a history of } \\
\text { antiplatelet use is } \\
\text { uncertain }\end{array}$ & $\begin{array}{l}\text { Not dialyzable } \\
\text { DDAVP } 0.4 \mu \mathrm{g} / \\
\mathrm{kg}\end{array}$ & $\begin{array}{l}\text { DDAVP } 0.4 \mu \mathrm{g} / \mathrm{kg} \text { IV (Conditional } \\
\text { recommendation, low-quality evidence) [90] } \\
\text { Platelet transfusion is not recommended } \\
\text { (Conditional recommendation, low-quality } \\
\text { evidence) [90] } \\
\text { Platelet transfusion for patients who will } \\
\text { undergo a neurosurgical procedure } \\
\text { (Conditional recommendation, moderate } \\
\text { quality of evidence) [90] } \\
\text { DDAVP can be used in addition to platelet } \\
\text { transfusion in patients who will undergo } \\
\text { neurosurgical procedure (Conditional } \\
\text { recommendation, low-quality evidence) [90] }\end{array}$ \\
\hline Ticlopidine & $\begin{array}{l}\text { Irreversible inhibition of } \\
\text { P2Y12 ADP receptor }\end{array}$ & $\begin{array}{l}60 \% \text { renal } \\
23 \% \text { fecal } \\
12 \text { hours } \\
\text { (increases with RF } \\
\text { to } 4-5 \text { days after } \\
\text { repeated doses) }\end{array}$ & $\begin{array}{l}\text { It is unclear if anti } \\
\text { platelet therapy } \\
\text { increases the } \\
\text { incidence of ICH }\end{array}$ & $\begin{array}{l}\text { Light Transmission } \\
\text { Platelet } \\
\text { Aggregation with } \\
\text { or without } \\
\text { Secretion }\end{array}$ & $\begin{array}{l}\text { None } \\
\text { The usefulness of platelet } \\
\text { transfusions in ICH patients } \\
\text { with a history of } \\
\text { antiplatelet use is } \\
\text { uncertain }\end{array}$ & $\begin{array}{l}\text { Not dialyzable } \\
\text { DDAVP } 0.4 \mu \mathrm{g} / \\
\mathrm{kg}\end{array}$ & $\begin{array}{l}\text { DDAVP } 0.4 \mu \mathrm{g} / \mathrm{kg} \text { IV (Conditional } \\
\text { recommendation, low-quality evidence) [90] } \\
\text { Platelet transfusion is not recommended } \\
\text { (Conditional recommendation, low-quality } \\
\text { evidence) [90] } \\
\text { Platelet transfusion for patients who will } \\
\text { undergo a neurosurgical. procedure } \\
\text { (Conditional recommendation, moderate } \\
\text { quality of evidence) [90] } \\
\text { DDAVP can be used in addition to platelet } \\
\text { transfusion in patients who will undergo } \\
\text { neurosurgical procedure (Conditional } \\
\text { recommendation, low-quality evidence) [90] }\end{array}$ \\
\hline Dipyridamole & $\begin{array}{l}\text { Reversible adenosine } \\
\text { reuptake inhibitor }\end{array}$ & $\begin{array}{l}\text { Fecal } \\
10 \text { hours }\end{array}$ & $\begin{array}{l}\text { It is unclear if anti } \\
\text { platelet therapy } \\
\text { increases the } \\
\text { incidence of } \mathrm{ICH}\end{array}$ & $\begin{array}{l}\text { Light Transmission } \\
\text { Platelet } \\
\text { Aggregation with } \\
\text { or without } \\
\text { Secretion }\end{array}$ & $\begin{array}{l}\text { None } \\
\text { The usefulness of platelet } \\
\text { transfusions in ICH patients } \\
\text { with a history of } \\
\text { antiplatelet use is } \\
\text { uncertain }\end{array}$ & $\begin{array}{l}\text { Not dialyzable } \\
\text { DDAVP } 0.4 \mu \mathrm{g} / \\
\text { kg }\end{array}$ & $\begin{array}{l}\text { DDAVP } 0.4 \mathrm{mcg} / \mathrm{kg} \text { IV (Conditional } \\
\text { recommendation, low-quality evidence) [90] } \\
\text { Platelet transfusion is not recommended } \\
\text { (Conditional recommendation, low-quality } \\
\text { evidence) [90] } \\
\text { Platelet transfusion for patients who will } \\
\text { undergo a neurosurgical procedure }\end{array}$ \\
\hline
\end{tabular}

neurosurgical procedure (Conditional

recommendation, low-quality evidence) [90] 
Table 3 Anticoagulants and reversal strategies (Continued)

\begin{tabular}{|c|c|c|c|c|c|c|c|}
\hline & & & & & & & recommendation, low-quality evidence) [90] \\
\hline Cilostazol & $\begin{array}{l}\text { Reversible } \\
\text { phosphodiesterase III } \\
\text { inhibitor, increases } \\
\text { cAMP, inhibits ADP- } \\
\text { induced platelet aggre- } \\
\text { gation, and causes } \\
\text { vasodilation }\end{array}$ & $\begin{array}{l}74 \% \text { renal } \\
20 \% \text { fecal } \\
10 \text { hours }\end{array}$ & $\begin{array}{l}\text { It is unclear if anti } \\
\text { platelet therapy } \\
\text { increases the } \\
\text { incidence of ICH }\end{array}$ & $\begin{array}{l}\text { Light Transmission } \\
\text { Platelet } \\
\text { Aggregation with } \\
\text { or without } \\
\text { Secretion }\end{array}$ & $\begin{array}{l}\text { None } \\
\text { The usefulness of platelet } \\
\text { transfusions in ICH patients } \\
\text { with a history of } \\
\text { antiplatelet use is } \\
\text { uncertain }\end{array}$ & $\begin{array}{l}\text { Not dialyzable } \\
\text { DDAVP } 0.4 \mathrm{\mu g} / \\
\mathrm{kg} \\
\text { The usefulness } \\
\text { of platelet } \\
\text { transfusions in } \\
\mathrm{ICH} \text { patients } \\
\text { with a history of } \\
\text { antiplatelet use } \\
\text { is uncertain }\end{array}$ & $\begin{array}{l}\text { DDAVP } 0.4 \mu \mathrm{\mu g} / \mathrm{kg} \text { IV (Conditional } \\
\text { recommendation, low-quality evidence) [90] } \\
\text { Platelet transfusion is not recommended } \\
\text { (Conditional recommendation, low-quality } \\
\text { evidence) [90] } \\
\text { Platelet transfusion for patients who will } \\
\text { undergo a neurosurgical procedure } \\
\text { (Conditional recommendation, moderate } \\
\text { quality of evidence) [90] } \\
\text { DDAVP can be used in addition to platelet } \\
\text { transfusion in patients who will undergo } \\
\text { neurosurgical procedure (Conditional } \\
\text { recommendation, low-quality evidence) [90] }\end{array}$ \\
\hline
\end{tabular}

Not dialyzable (26\% renal 7 hours (metabolite increases the Platelet The usefulness of platelet DDAVP $0.4 \mu \mathrm{g} /$ 9 hours) or withou Secretion with a history of uncertain

P $\angle M W H$ low-molecular-weight heparin, UFH unfractionated heparin, $R F$ renal failure, GFR glomerular filtration rate, BID two times a day, SDH subdural hematoma, SAH subarachnoid hemorrhage, $H R$ heartrate, ACT acti-
vated clotting time, FFP Fresh frozen plasma, $I V$ intravenous, VKA vitamin K antagonist, rFVIlla recombinant activated factor VII, SC subcutaneous, DDAVP Desmopressin undergo a neurosurgical procedure
(Conditional recommendation, moderate quality of evidence) [90]

DDAVP can be used in addition to platelet transfusion in patients who will undergo recommendation, low-quality evidence) [90] DDAVP $0.4 \mathrm{mcg} / \mathrm{kg}$ IV (Conditional

recommendation, low-quality evidence) [90] (Conditional recommendation, low-quality evidence) [90]

Platelet transfusion for patients who will

Conditional recommendation, moderate quality of evidence) [90]

DDAVP can be used in addition to platelet transfusion in patients who will undergo neurosurgical procedure (Conditional

recommendation, low-quality evidence) $[90]$

Platelet transfusion is not recommended

evidence) [90]

recommendation, moderate

transfusion in patients who will undergo neurosurgical procedure (Condition 
administered and it is usually adjusted by weight and/ or INR, although fixed doses of at least $20 \mathrm{UI} / \mathrm{kg}$ of factor IX have been reported to be effective [91]. Four-factor PCC might be more effective in reversing warfarin effect than three-factor PCC $[92,93]$. A list of some four-factor PCC products available in Canada, the USA, and Europe is presented in Additional file 1. PCCs are effective for rapid reversal of anticoagulation due to warfarin use and are considered the first-choice option in some guidelines [94, 95]. Hickey et al. [96] showed in a retrospective cohort study that the median time for INR reversal was significantly shorter with four-factor PCC when compared with FFP (5.7 hours vs 11.8 hours, respectively, $p<0.0001$ ). Additionally, FFP was associated with higher incidence of serious adverse events, especially heart failure (19.5\% vs $9.7 \%, p=0.014$; relative risk $2.0,95 \% \mathrm{CI}$ 1.1-3.5). In a phase IIIb study, Sarode et al. randomized 202 patients with active warfarin-related major bleeding to four-factor PCC or FFP. Rapid INR reduction, defined as the INR correction $(\leq 1.3)$ at 0.5 hours after the end of infusion, occurred in $62.2 \%$ of cases with four-factor PCC and only in $9.6 \%$ of patients who received FFP transfusion [97]. Although the use of recombinant activated factor VII (rFVIIa) has been described as a possible option for warfarin-related coagulopathy reversal, rFVIIa does not replace the levels of other vitamin Kdependent factors and has a short half-life. Current guidelines therefore do not recommend its use for reversal of warfarin-related $\mathrm{ICH}$. Additionally, it is 15 times more expensive than FFP and at least 3.5 times more expensive than PCC, and is associated with a higher risk of INR rebound [98]. In summary, patients with warfarin-related ICH and elevated INR should have vitamin $\mathrm{K}$ antagonist withheld, and should receive vitamin $\mathrm{K}$ (10 mg IV over 30 minutes) concomitantly with PCC guided by INR level or weighted-base dosing $(20 \mathrm{UI} / \mathrm{kg})$. If $\mathrm{PCC}$ is not available, FFP should be administered in a dose of $10-40 \mathrm{ml} / \mathrm{kg}$. rFVIIa is not recommended in this clinical scenario.

b. Unfractionated heparin (UFH) and low molecular weight heparins (LMWHs). UFH prevents fibrin formation by indirectly inhibiting factors Xa and IIa (thrombin) through the activation of antithrombin. Heparin-related $\mathrm{ICH}$ occurs in approximately $0.1-0.2 \%$ of patients on continuous infusion of UFH for a non-neurological indication. For patients who develop $\mathrm{ICH}$ while on UFH infusion, heparin reversal is warranted. Heparin infusion should be immediately interrupted and protamine sulfate should be administered at a dose of $1 \mathrm{mg}$ for every 100 units of heparin given in the previous 2-3 hours (maximum single dose of $50 \mathrm{mg}$ ). A repeat dose of $0.5 \mathrm{mg}$ of protamine per 100 units of UFH may be given if the aPTT remains elevated [90]. Similarly to UFH, LMWHs also bind and activate antithrombin but have less effect on thrombin compared with UFH, although approximately the same effect on factor Xa. Protamine appears to only partially neutralize the anti-factor Xa activity of LMWH and therefore, in case of need for reversal, this cannot be done completely. The main goal of LMWH reversal is discontinuation of the drug, although protamine use can be attempted. For enoxaparin given within 8 hours, protamine sulfate should be administered at the dose of $1 \mathrm{mg}$ per $1 \mathrm{mg}$ of enoxaparin (maximum single dose of $50 \mathrm{mg}$ ); for enoxaparin given within 8 and 12 hours, a dose of $0.5 \mathrm{mg}$ of protamine per $1 \mathrm{mg}$ of enoxaparin should be administered. Beyond 12 hours of enoxaparin administration, protamine administration is not suggested. For dalteparin or nadroparin, protamine sulfate should be given at a dose of $1 \mathrm{mg}$ per $100 \mathrm{IU}$ of dalteparin/nadroparin administered in the past 3-5 half-lives (maximum single dose of $50 \mathrm{mg})$. Factor VIIa $(90 \mu \mathrm{g} / \mathrm{kg})$ can be used if protamine is contraindicated or in LMWH-related ICHs that are refractory to protamine [90].

c. Antiplatelet agents. The literature on APT and its association with outcomes after ICH is still controversial. Several reports described the association of antiplatelet agent use with hematoma expansion and worse clinical outcome, including increased mortality rate [99-101]. However, more recent studies reported that patients on antiplatelet agents prior to $\mathrm{ICH}$ have similar rates of hematoma expansion compared with patients not receiving these agents, and the functional outcome may be independent of antiplatelet use [102-104]. For example, in the Cerebral Hematoma and NXY-059 Treatment (CHANT) trial, a secondary analysis of the placebo arm found no association between APT use and ICH outcomes [102]. Because it remains controversial whether APT influences hematoma expansion or functional outcome, the clinical utility of its reversal is uncertain [105]. In patients with $\mathrm{ICH}$ and on APT, the agent should be discontinued immediately. Some observational studies suggested potential benefit from platelet transfusion [106]. Recently, Baharoglu et al. [107] published the results of the PATCH study, the first randomized, open-label, phase 3 trial investigating the effect of platelet transfusion in patients with spontaneous $\mathrm{ICH}$, who were receiving APT. The study included 190 participants from the Netherlands, the UK, and France. The study showed increased odds of a 
shift towards death or dependence at 3 months in the group of patients receiving platelet transfusion compared with the standard care group (adjusted common OR 2.5, 95 \% CI 1.18-3.56, $p=0.0114$ ). Secondly, more serious adverse events were reported in patients who received platelet transfusion (42\%), compared with $29 \%$ in patients who received standard care alone. Interestingly, the two groups showed a difference in hematoma volume, although this was not significant (13.1 (5.4-42.4) $\mathrm{ml}$ in the intervention group vs $8.0(4.4-25.8) \mathrm{ml}$ in the standard of care group). In the post-hoc analysis the primary outcome remained unchanged when adjusted for ICH volume at baseline, but there was an apparent potential benefit for platelet transfusion as the hematoma volume increased; however, the very small size of the population precluded a better-powered exploratory analysis. Until the results of another similar randomized trial (ClinicalTrials.gov NCT00699621) are available, platelet transfusion should be considered not beneficial but rather potentially harmful for people taking APT and such decisions should be considered on an individual basis [9]. For example, many authors recommend platelet transfusion only for patients with aspirin-associated or ADP inhibitor associated $\mathrm{ICH}$ and for whom an emergency neurosurgical procedure is planned [108].

A recent pilot study evaluated the role of desmopressin (DDAVP) to improve platelet function in patients with $\mathrm{ICH}$ and reduced platelet activity or on aspirin therapy [109]. Desmopressin $(0.4 \mu \mathrm{g} / \mathrm{kg}$ IV given over 30 minutes) increased platelet activity, as measured by von Willebrand factor antigen and closure times (PFA-100 with epinephrine), 1 hour after DDAVP administration. The DDAVP effect may be short lived, however, and platelet function abnormalities may return within 3 hours [110]. Based on these limited data, a single dose of DDAVP (0.4 $\mu \mathrm{g} / \mathrm{kg}$ IV given over 30 minutes) may be considered for patients with aspirin-associated, COX1 inhibitor-associated, or ADP receptor inhibitorassociated ICH.

\section{d. New oral anticoagulants}

- Factor Xa inhibitors. These oral anticoagulant agents (e.g., rivaroxaban, apixaban, and edoxaban) act as direct factor Xa inhibitors and prevent factor Xa-dependent conversion of prothrombin to thrombin. Current indications include primary stroke prevention (i.e., in nonvalvular atrial fibrillation), treatment of deep vein thrombosis and pulmonary embolism, and secondary prevention of venous thromboembolism (VTE) $[108,111]$. Compared with warfarin, factor Xa inhibitors have shown a lower risk of $\mathrm{ICH}$ (Table 3). However, currently there is no specific antidote commercially available for this class of drug, and most information on their reversal is limited to ex-vivo and in-vivo studies on healthy volunteers and animal models of bleeding. Current recommendations suggest immediate discontinuation of the drug followed by the administration of four-factor PCC (50 U/kg) or activated PCC $(50 \mathrm{U} / \mathrm{kg})$ in the case of patients presenting within 3-5 terminal half-lives of the drug or in the presence of liver failure. In case of recent ingestion (within 2 hours), $50 \mathrm{~g}$ of activated charcoal is recommended [108].

- Direct thrombin inhibitor reversal. Available direct thrombin inhibitors include competitive direct thrombin inhibitor (e.g., oral dabigatran), reversible direct thrombin inhibitors (argatroban and bivalirudin, both intravenous only), and irreversible direct thrombin inhibitors (e.g., desirudin SC and lepirudin IV). Their main current indications include primary stroke prevention in patients with non-valvular AF, treatment of VTE, and management of heparin-induced thrombocytopenia [108]. Data on the incidence and outcomes of direct thrombin inhibitor-related $\mathrm{ICH}$ is scant. Dabigatran seems to be associated with an $\mathrm{ICH}$ rate of $0.2-0.3 \%$ /year, which is lower compared with warfarin [108]. Recently, a dabigatran-specific monoclonal antibody, idarucizumab (Praxbind ${ }^{\circledR}$ ), has been approved for clinical use. The use of idarucizumab in dabigatran reversal was demonstrated in an interim analysis of the Reversal Effects of Idarucizumab on Active Dabigatran Study (the RE-VERSE AD study) [112]. The report included 90 patients (one-third with $\mathrm{ICH}$ ) with uncontrolled or life-threatening bleeding, or requiring emergency surgical procedures ( $<8$ hours) [112]. Patients received $5 \mathrm{~g}$ of idarucizumab divided in two doses of $2.5 \mathrm{~g}$ at 15 -minute intervals. Idarucizumab reversed anticoagulation in $90 \%$ of patients within 10-30 minutes of drug infusion, as assessed by the dilute thrombin time (TT) and ecarin clotting time (ECT). Therefore, for patients with dabigatran-related $\mathrm{ICH}$, emergency treatment should include discontinuation of the drug, followed by two doses of $2.5 \mathrm{~g}$ idarucizumab IV at 15-minute intervals, if the last dose of dabigatran was ingested within 3-5 half-lives or if renal failure is present. In the occurrence of dabigatran intoxication or renal failure, the use of hemodialysis can be considered. If idarucizumab is not available, or if the $\mathrm{ICH}$ is related to other direct thrombin 
inhibitors, emergency treatment should include discontinuation of the drug followed by the administration of activated PCC $(50 \mathrm{U} / \mathrm{kg})$ or a four-factor PCC $(50 \mathrm{U} / \mathrm{kg})$, if the last dose of drug was ingested within 3-5 terminal half-lives. If the drug was taken more than 3-5 half-lives before presentation, reversal in not indicated. In the case of recent ingestion (within 2 hours), $50 \mathrm{~g}$ of activated charcoal is recommended. The use of rFVIIa or FFP in direct thrombin inhibitor-related intracranial hemorrhage is not recommended.

e. rFVIIa in patients without coagulopathy. The use of rFVIIa in noncoagulopathic patients has been studied in multiple randomized trials and has been shown to reduce hematoma growth but not to improve patient survival or functional outcome. Additionally, its use is associated with an increased rate of arterial thromboembolic adverse events [113]. Currently, two clinical trials-the SPOTLIGHT trial (ClinicalTrials.gov NCT01359202) and the STOP-IT trial (ClinicalTrial.gov NCT0081088) - are underway to clarify the role of rFVIIa in ICH patients with a positive-spot sign. Current guidelines do not recommend the use of rFVIIa in unselected noncoagulopathic ICH patients [9].

f. Other anticoagulant agent reversal strategies are summarized in Table 3.

Table 4 summarizes recent studies examining hemostatic therapy, blood pressure management, and the surgical approach to $\mathrm{ICH}$.

\section{Surgical treatment of spontaneous intracranial hemorrhage}

\section{Supra-tentorial hemorrhage}

The benefits of clot removal have been addressed in two randomized trials. The Surgical Trial in Intracerebral Hemorrhage (STICH) randomized 1033 patients with supra-tentorial hemorrhage (lobar or ganglionic hematoma) to early surgery (within 96 hours of ictus) versus standard of care (i.e., medical management with delayed surgery if necessary) [114]. No difference in favorable functional outcome at 6 months was found $(p=0.414)$. However, the subgroup of patients with superficial ICHs (lobar hemorrhage within $1 \mathrm{~cm}$ of the cortical surface) who underwent surgery had better outcomes. This result prompted a second trial, STICH II, aiming at randomizing patients with superficial lobar hematomas (10-100 ml) to early surgery versus medical management with delayed surgery if necessary [115]. Patients with IVH or coma were excluded. STICH II found no difference in mortality or severe disability with early surgery $(p=0.37)$. Of note, patients with predicted poor prognosis at enrollment (estimated according to a prognostic model taking into account GCS, age, and $\mathrm{ICH}$ volumes: $10 \times \mathrm{GCS}-$ age $-0.64 \times$ volume) were more likely to have a favorable outcome with early surgery than with initial conservative treatment (OR 0.49, $p=0.02$ ). Such a benefit with early surgery was not detected in the group of patients with predicted good prognosis at enrollment (OR 1.12, $p=0.57$ ) [115].

\section{Posterior fossa hemorrhage}

Hemorrhage involving the posterior fossa (cerebellum or brainstem; Fig. 2) can be associated with life-threatening complications, such as acute hydrocephalus secondary to fourth-ventricle compression and direct brainstem compression and/or herniation through the foramen magnum. Treatment strategies include posterior fossa (suboccipital) decompressive craniectomy, external ventricular drain (EVD) insertion or conservative management. There is no randomized trial addressing the best approach or timing to manage infra-tentorial hemorrhage and the evidence available is based on class III studies. Different protocols and algorithms have been published, directing management strategies on the basis of GCS and hematoma size [116], degree of fourth-ventricle compression [117], or GCS and presence of hydrocephalus [118]. Patients with a GCS score of 14-15 and small hematomas $(\leq 3 \mathrm{~cm})$ can be treated conservatively. In the case of neurological deterioration, hematoma drainage \pm craniectomy should be strongly considered. In comatose patients without brainstem reflexes, formal neurological determination of death should be considered. Comatose patients with preserved brainstem reflexes should be considered for emergency hematoma drainage and suboccipital decompressive craniectomy. Insertion of EVD alone for treatment of cerebellar occupying lesions remains controversial because of the theoretical risk of upward herniation and is not recommended by the AHA guidelines [9]. However, management of recent cohorts of patients with cerebellar infarcts showed that EVD alone is a possible treatment, and can reduce the need for suboccipital decompressive craniectomy [119].

\section{Intraventricular hemorrhage}

IVH occurs in nearly half of ICH patients. Isolated IVH (primary IVH) occurs rarely but more often is the result of secondary extension of a parenchymal hematoma into the ventricular system. The presence of blood in the ventricles can interrupt the normal cerebrospinal fluid (CSF) flow and cause obstructive (noncommunicating) hydrocephalus and increased ICP. Placement of an EVD to drain CSF and monitor ICP should therefore be considered in patients with acute hydrocephalus/IVH and GCS $\leq 8$ or with signs of transtentorial herniation [9]. A practical issue arises from the clot burden in the ventricular system and the frequent obstruction of ventricular drain. Techniques such as neuroendoscopy or intraventricular thrombolysis (IVT) have been 
Blood pressure management

Rapid Blood Pressure Reduction in Acute Intracerebral Hemorrhage [152]

INTERACT trial [76]

ATACH I trial [154]
Prospective randomized, single-center study Primary outcome: clinical deterioration (NIHSS drop $\geq 2$ points) within the first 48 hours. Hematoma enlargement at 24 hours was a secondary endpoint

Eligible patients: acute spontaneous supratentorial ICH within 8 hours of symptom onset Exclusion criteria: history of head trauma; coma with signs of herniation; coagulopathy; $\mathrm{MAP}<110 \mathrm{mmHg}$ at presentation; secondary $\mathrm{ICH}$; surgical hematoma evacuation

Open-label, multicenter, blinded outcome, randomized trial; 44 hospital sites in Australia, China, and South Korea

Primary outcome: proportional change in hematoma volume at 24 hours

Eligible patients: spontaneous ICH and elevated BP ( $\geq 2$ measurements of $150-220 \mathrm{mmHg}$ recorded $\geq 2$ minutes apart)

Exclusion criteria: SBP $>220 \mathrm{mmHg}$ or hypertensive encephalopathy; severe cerebral artery stenosis or renal failure; secondary $\mathrm{ICH}$ or the use of a thrombolytic agent; ischemic stroke within 30 days; GCS 3-5; significant prestroke disability or medical illness; or early planned decompressive neurosurgical intervention

Phase I, dose-escalation, multicenter prospective

study
Eligible patients: spontaneous ICH with admission Eligible patients: spontaneous $\mathrm{CH}$ with admission
$\mathrm{SBP} \geq 170 \mathrm{mmHg}$ on two repeat measurements at least 5 minutes apart. Symptom onset < 6 hours at the time to evaluation and initiation of treatment with IV nicardipine

Primary outcomes: (1) Feasibility end point: SBP reduction and maintenance in the respective target range achieved (treatment success) or not (failure): (2) Safety end points: (a) neurologic deterioration (defined by a decline in the GCS
Blood pressure targets: standard treatment $(\mathrm{MAP}=110-130 \mathrm{mmHg})$ vs aggressive $\mathrm{BP}$ treatment (MAP $<110 \mathrm{mmHg}$ )

Maximum interval from symptom onset to treatment: 8 hours

Duration of treatment: 48 hours

Drugs used:

- Initially, intermittent labetalol infusions (10-20 mg)

- If target blood pressure was not achieved, a continuous infusion of nicardipine $(5-15 \mathrm{mg} /$ hour) was started

- More severe cases were treated with intravenous nicardipine from the onset (i.e, initial dose, $5 \mathrm{mg} /$ hour followed by titration and increases of $2.5 \mathrm{mg} /$ hour every 5-15 minutes, and no bolus)

- Most severe cases of hypertension were treated with sodium nitroprusside infusion at $0.3 \mu \mathrm{g} / \mathrm{kg} /$ minute IV infusion and titrated every few minutes to desired effect

Blood pressure targets: early intensive BPlowering strategy (target SBP $=140 \mathrm{mmHg}$ within 1 hour) vs standard approach (target $\mathrm{SBP}=180 \mathrm{mmHg}$ )

Maximum interval from symptom onset to treatment: 6 hours

Duration of treatment: 7 days

Drugs used: treatment conducted with locally available intravenous or oral BP-lowering agent, each included (treatment group): furosemide (35\%), urapidil (47\%), phentolamine (16\%), glycerol trinitrate $(10 \%)$, labetalol (6\%), nicardipine (5\%), hydralazine (3 \%), metoprolol (1 \%), nitrate patch (3\%). It also included some oral agents

\section{Blood pressure targets:}

- Tier 1: SBP $\geq 170$ and $<200 \mathrm{mmHg}$

- Tier 2: SBP $\geq 140$ and $<170 \mathrm{mmHg}$

- Tier 3: SBP $\geq 110$ and $<140 \mathrm{mmHg}$

Maximum interval from symptom onset to treatment: 6 hours

Duration of treatment: 18-24 hours

Drugs used: intravenous nicardipine infusion,

initiated at $5 \mathrm{mg} /$ hour, and then increased by

$2.5 \mathrm{mg}$ every 15 minutes as needed, up to a maximum of $15 \mathrm{mg}$ /hour. Once the target SBP was achieved, the infusion rate was decreased
21 patients in each group

Treatment was started on average $3.2 \pm 2.2$ hours after symptom onset

Target blood pressure was achieved within $87.1 \pm 59.6$ minutes in the standard group and $163.5 \pm 163.8$ minutes in the aggressive BP treatment group

No significant differences in early neurologica deterioration, hematoma and edema growth, and clinical outcome at 90 days (mRS)

203 patients randomized to intensive BP management vs 201 to standard guidelinesbased management

Trend toward lower hematoma growth at 24 hours in the intensive treatment group (difference 22.6\%, $95 \% \mathrm{Cl} 0.6-44.5 \%, p=0.04$; absolute difference in volume $1.7 \mathrm{ml}, 95 \% \mathrm{Cl}$ $0.5-3.9 \mathrm{ml}, p=0.13$ )

No excess of neurological deterioration, other clinical outcomes, or adverse events

60 patients enrolled (Tier $1=18$; Tier $2=20$; Tier $3=22$ )

Nine patients in Tier 3 had treatment failure Seven patients had neurologic deterioration (one, two, and four in Tiers 1, 2, and 3 , respectively)

One subject in Tier 2 and three in Tier 3 had serious adverse events; however, the safetystopping rule was not activated in any of the tiers

These results confirmed the feasibility and safety of early rapid lowering of BP in ICH and formed 
Table 4 Evidence-based summary (Continued)

score 2 or increase in NIHSS score 4 points not explained by use of sedatives or hypnotics) within 24 hours from treatment initiation; (b) serious adverse events within 72 hours

ICH ADAPT study [74]

Multicenter, prospective, randomized, openlabel, with blinded evaluation study. A block randomization design (six patients/block) stratified by onset to treatment time ( $\leq 6$ and 6-24 hours)

Eligible patients: spontaneous ICH diagnosed $<24$ hours after onset and SBP $>150 \mathrm{mmHg}$ Exclusion criteria: Secondary ICH (e.g., vascular malformation), planned surgical resection, or contraindications to $C T$ perfusion (CTP. e. contrast allergy or renal impairment)

Primary end-point: difference in perihematoma relative cerebral blood flow (CBF) between treatment groups as assessed by CT perfusion imaging 2 hours post randomization

\section{INTERACT2 trial [71]}

ATACH 2 [77]
International, multicenter, prospective, randomized, open-treatment, blinded end-point trial

Eligible patients: spontaneous ICH and elevated BP (at least two SBP measurements of $\geq 150$ and $\leq 220 \mathrm{mmHg}$, recorded 2 or more minutes apart)

Exclusion criteria: structural cerebral cause for the intracerebral hemorrhage; GCS 3-5; massive hematoma with a poor prognosis; or early planned surgery to evacuate the hematoma Primary outcome: death or major disability (mRS3-6 at 90 days)

International, multicenter, randomized, open label trial

Eligible patients: ICH (volume, $<60 \mathrm{~cm}^{3}$ ) and Eligible pati

Exclusion criteria: structural cerebral cause for the intracerebral hemorrhage; GCS 3-4; massive hematoma with a poor prognosis; or early planned surgery to evacuate the hematoma Primary outcome: death or major disability (mRS 4-6 at 3 months) by 1-3 mg/hour. If the SBP dropped below the specified levels, infusion was reduced by

$2.5 \mathrm{mg} /$ hour every 15 minutes until the drug was stopped

Blood pressure targets: SBP target $<150 \mathrm{mmHg}$ vs $<180 \mathrm{mmHg}$ to be achieved within 1 hour of randomization

Drugs used: labetalol test dose: $10 \mathrm{mg}$ bolus over 1 minute. If SBP $>$ target (150 or $180 \mathrm{mmHg}$ ) and heart rate (HR) > $55 \mathrm{bpm}$, the bolus was repeated (10 $\mathrm{mg}$ bolus in 5 minutes). 10-20 mg IV push every 5 minutes until $\mathrm{SBP}<$ target or $\mathrm{HR}<55$. Labetalol maximum $300 \mathrm{mg} / 24$ hours

Hydralazine was used only if SBP persistently $>$ target, or if HR was below 55

Enalapril was used in the SBP $<150 \mathrm{mmHg}$ target group. It was considered in patients with labile $\mathrm{BP}$, who required repeated doses of labetalol and/or hydralazine. $1.25 \mathrm{mg}$ IV every 6 hours PRN

Blood pressure targets: intensive treatment (SBP $<140 \mathrm{mmHg}$ within 1 hour) vs guidelinerecommended treatment (SBP $<180 \mathrm{mmHg}$ ) Maximum interval from symptom onset to

treatment: 6 hours

Duration of treatment: 7 days

Drugs used: treatment conducted with locally available intravenous BP-lowering agent, each included (treatment group): alpha-adrenergic antagonist (i.e.,

urapidil, $32.5 \%$ ), calcium-channel blocker (nicardipine or nimodipine, $16.2 \%$ ), combined alphaand beta-blocker (labetalol, $14.4 \%$ ), nitroglycerin (14.9\%),

furosemide (12.4\%), nitroprusside (12.1\%), hydralazine $(5.9 \%)$

Blood pressure targets: intensive treatment (SBP $110-139 \mathrm{mmHg}$ within 2 hours) vs SBP between 140 and $179 \mathrm{mmHg}$ )

Maximum interval from symptoms onset to treatment: 4.5 hours

Duration of treatment: 24 hours

Drugs used: nicardipine IV, started at a dose of $5 \mathrm{mg}$ per hour, and increased by $2.5 \mathrm{mg}$ per hour every 15 minutes (maximum dose of

$15 \mathrm{mg}$ per hour). Intravenous labetalol was the based for the larger randomized ATACH II trial

75 patients enrolled

Focal decreases in CBF and cerebral blood volume within the perihematoma region evident in all patients.

After adjustment for baseline intraparenchymal hematoma volume and time to randomization, perihematoma relative $\mathrm{CBF}$ not significantly lower in patients randomized to SBP $<150 \mathrm{mmHg}(p=0.18$; absolute difference $0.03,95 \% \mathrm{Cl}-0.018$ to 0.078

2839 patients enrolled at 144 hospitals in 2 countries ( $n=1403$ early intensive treatment; $n=1436$ guideline-recommended treatment) No statistically significant difference between two groups in the rates of death or severe disability ( $52 \%$ vs $55.6 \%$ OR with intensive disability $(52 \%$ vs treatment 0.87 , $95 \%$ Cl $0.75-1.01, p=0.06$ ) A pre-specified ordinal analysis of the mRS score showed significantly lower scores with intensive treatment (OR for greater disability $0.87,95 \% \mathrm{Cl}$ $0.77-1.00, p=0.04$

1000 patients enrolled at 110 sites in six countries $(n=500$ intensive treatment; $n=500$ standard treatment)

No statistically significant difference in the rates of death or disability (38.7 vs $37.7 \%$; intensive vs standard treatment, respectively)

Relative risk, 1.04; 95 \% Cl 0.85-1.27; analysis was adjusted for age, initial GCS score, and presence or absence of intraventricular hemorrhage 
added as second-line agent, if the systolic blood pressure target was not reached

Intensive blood pressure reduction in acute intracerebral hemorrhage: a metaanalysis [155]

Hemostasis

FAST trial [153]

A meta-analysis of the efficacy and safety of recombinant activated factor VII for patients with hemorrhage without hemophilia [131]

- ICH ADAPT [74]
Systematic review and meta-analysis according to PRISMA guidelines. Included available randomized controlled trials that randomized patients with acute $\mathrm{ICH}$ to either intensive or guideline BP-reduction protocols at the time of publication

Included four studies:

- Rapid Blood Pressure Reduction in Acute

Intracerebral Hemorrhage [152]

- INTERACT [76]

- INTERACT2 [71]

Multicenter, randomized, double blinded,

placebo-controlled trial

Eligible patients: spontaneous ICH within 3 hours of symptom onset

Exclusion criteria: GCS $\leq 5$; secondary $\mathrm{ICH}$; known use of anticoagulant therapy,

thrombocytopenia, or coagulopathy; acute sepsis; crush injury; disseminated intravascular coagulation; pregnancy; previous disability;

known recent thromboembolic disease

Primary outcome: death or severe disability (mRS $5-6$ at 90 days)

Systematic review and meta-analysis

Included five studies (one included traumatic ICH patients):

- Safety and feasibility of recombinant factor

VIla for acute intracerebral hemorrhage

- Recombinant activated factor VII for acute intracerebral hemorrhage

- Recombinant activated factor VII for acute intracerebral hemorrhage: US phase IIA tria

- Recombinant factor VIIA in traumatic

intracerebral hemorrhage: results of a doseescalation clinical trial

Efficacy and safety of recombinant activated factor VII for acute intracerebral hemorrhage

Platelet transfusion (leukocyte-depleted, either

Multicenter, randomized, open-label, masked end-point, parallel-group, phase 3 trial; 60 hospitals (36 Netherlands, 13 UK, 11 France)
The rate of renal adverse events within 7 days was significantly higher in the intensive treatment group ( $9.0 \%$ vs $4.0 \%, p=0.002$ )

3315 patients

Death rates were similar between the groups (OR $51.01,95 \%$ Cl $0.83-1.23, p \leq 0.914$ ) Intensive BP-lowering treatment tended to be associated with lower 3-month death or dependency (mRS grades 3-6) compared with guideline treatment (OR 5 0.87, $95 \%$ Cl 0.76 $1.01, p=0.062$

No evidence of heterogeneity between estimates $\left(R^{2} \leq 0 \%, p \leq 0.723\right)$, or publication bias in the funnel plots ( $p \leq 0.993$, Egger statistical test), was detected

Intensive BP reduction was also associated with a greater attenuation of absolute hematoma growth at 24 hours (standardized mean difference \pm SE: $-0.110 \pm 0.053, p=0.038$ )

841 patients $(n=268$, placebo; $n=276, \mathrm{rFV}$ $20 \mu \mathrm{g} / \mathrm{kg} ; n=297, \mathrm{rFV} \| \mathrm{ll} 80 \mu \mathrm{g} / \mathrm{kg}$ )

$80 \mu \mathrm{g} / \mathrm{kg}$ of $\mathrm{rFVIla}$ associated with significant reduction in ICH expansion (mean estimated increase in volume of ICH: $26 \%$ placebo; $18 \%$ $20 \mu \mathrm{g} / \mathrm{kg} ; 11 \% 80 \mu \mathrm{g} / \mathrm{kg}$ )

Despite the reduction in bleeding, no significant difference in the proportion of patients with poor outcome (24\% placebo; $26 \% 20 \mu \mathrm{g} / \mathrm{kg}$; $29 \% 80 \mu \mathrm{g} / \mathrm{kg}$ )

More arterial thromboembolic events in the group receiving $\mathrm{rFVII} 80 \mathrm{\mu g} / \mathrm{kg}$ versus placebo (9 \% vs $4 \%, p=0.04$ )

rFVlla reduced the change in $\mathrm{ICH}$ volume

There was no significant difference in mortality, mRS score or extended Glasgow Outcome Scale (GOS-E) score in patients treated with rFVIla or placebo

There was a significant increase in arterial thromboembolic adverse events in patients treated with rFVlla

There was an increase in deep vein thrombosis in patients with spontaneous $\mathrm{ICH}$ and traumatic $\mathrm{ICH}$

190 participants at 41 different sites enrolled between February 2009 and October 2015 (97 patients assigned to receive standard care with
PATCH trial [107] 
Table 4 Evidence-based summary (Continued)

Surgical treatment

STICH [114]

STICH II [115]
Eligible patients: nontraumatic supra-tentorial ICH; GCS 8-15; antiplatelet therapy for at least 7 days preceding $\mathrm{I} C H$; pre-ICH mRS 0-1 Exclusion criteria: epidural or subdural hematoma; underlying aneurysm or arteriovenous malformation; planned surgical evacuation of $\mathrm{ICH}$ within 24 hours of admission; IVH more than sedimentation in the posterior horns of the lateral ventricles; previous adverse reaction to platelet transfusion; vitamin $k$ antagonist use or knows coagulopathy thrombocytopenia $(<100,000$ cells/ml) Primary outcome: difference in functional outcome at 3 months after randomization scored with $\mathrm{mRS}$

International, multicenter, prospective, randomized trial

Eligible patients: spontaneous supra-tentorial ICH within 72 hours: uncertainty about the benefits of either treatment according to responsible neurosurgeon

Exclusion criteria: secondary $\mathrm{ICH}$; cerebellar hemorrhage or extension of a supra-tentorial hemorrhage into the brainstem; severe preexisting physical or mental disability or severe comorbidities; surgery not undertaken within 24 hours of randomization

Primary outcome: death or disability using the extended Glasgow Outcome Scale 6 months after ictus

International, multicenter, prospective, andomized, parallel group, pragmatic trial Eligible patients: spontaneous lobar ICH, $\leq 1 \mathrm{~cm}$ from the cortical surface of the brain, volume between 10 and $100 \mathrm{ml}$. within 48 hours of onset of ictus; best GCS motor score $\geq 5$ and best GCS eye score $\geq 2$

Exclusion criteria: secondary $\mathrm{I} C \mathrm{H}$; involvement of basal ganglia, thalamic, cerebellar, or brainstem be initiated within 6 hours of symptom onset and 90 minutes of brain imaging

- COX inhibitor, with or without adenosinereuptake inhibitor: 1 platelet concentrate (equivalent to 5 donor units) - ADP receptor inhibitor, with or without another antiplatelet drug: 2 platelet concentrates

Patients randomized to early surgery (hematoma evacuated within 24 hours of randomization by the method of choice of the responsible neurosurgeon, combined with the best medical treatment) or to initial conservative management (best medical treatment; later surgical evacuation allowed in case of neurological deterioration)

Patients randomized to early surgery (evacuation of hematoma within 12 hours of randomization) or initial conservative treatment (delayed evacuation permitted if judged clinically appropriate) platelet transfusion and 93 assigned to standard care without transfusion)

4 patients assigned to platelet transfusion did not receive it; 2 participants assigned to

standard care alone received platelet transfusion Primary outcome: higher odds of a shift towards death or dependence at 3 months in the

platelet transfusion group (adjusted common

OR 2.05, $95 \%$ Cl 1.18-3.56, $p=0.0114$ )

Secondary analysis: more patients in the platelet transfusion group with mRS 4-6 than those in

standard care group

n-hospital mortality: 24 (25\%) patients assigned to platelet transfusion; 15 (16\%) patients assigned to standard care alone

Pre-specified subgroup analyses (type of antiplatelet therapy; country; hematoma volume): no significant interaction

Serious adverse events: 40 (42\%) patients in platelet transfusion group; 28 (29\%) patients in standard care group

1033 patients from 83 centers in 27 countries $(n=503$ early surgery; $n=530$ initial conservative treatment)

Of the 468 patients randomized to early surgery analyzed at 6 months, $122(26 \%)$ had a

favorable outcome compared with 118 (24\%) of 496 patients randomized to initial conservative treatment (OR $0.89,95 \% \mathrm{Cl} 0.66-$ $1.19, p=0.414$; absolute benefit $2.3 \%$; relative benefit $10 \%)$

$26 \%$ of patients initially randomized to

conservative treatment underwent surgery after an initial period of observation

Subgroup analysis of patients with lobar ICH within $1 \mathrm{~cm}$ of the cortical surface who underwent surgery had a statistically significant increase in good outcomes compared with similar subjects in the medical arm (8 \% absolute increase, $p=0.02$ )

601 patients from 78 centers in 27 countries ( $n=307$ early surgery; $n=294$ initial conservative treatment)

Median time to craniotomy: 26 hours after stroke onset

No difference in the primary outcome (absolute difference $3.7 \%, 95 \% \mathrm{Cl}-4.3$ to $11.6 \%$; OR $0.86,95 \%$ Cl $0.62-1.20, p=0.37$ ) 
Table 4 Evidence-based summary (Continued)

regions; presence of IVH; severe pre-existing

In the subgroup of patients with a poor

physical or mental disability or severe

expected prognosis at enrollment (lower GCS,

comorbidities

greater age, and larger ICH volume), early

surgical intervention was associated with more

Primary outcome: prognosis-based favorable or

unfavorable outcome dichotomized from the

Extended Glasgow Outcome Scale at 6 months

after randomization

$p=0.02$ )

No advantage for surgery in the good

prognosis group (OR 1.2, $95 \%$ Cl 0.75-1.68,

$p=0.57)$

Among patients in the initial conservative

treatment group, $21 \%$ had surgery

BP blood pressure, CT computed tomography, GCS Glasgow Coma Scale, ICH intracerebral hemorrhage, mRS modified Rankin scale, NIHSS National Institutes of Health Stroke Scale, OR odds ratio, rFVIIa recombinant

activated factor VII, SBP systolic blood pressure, MAP mean arterial pressure, $I V$ intravenous, PRN when necessary 
investigated. The Clot Lysis Evaluation of Accelerated Resolution of Intraventricular Hemorrhage (CLEAR-IVH) trial demonstrated that the use of low-dose recombinant tissue plasminogen activator ( $r$-tPA) had an acceptable safety profile in patients with IVH, as well as being beneficial in accelerating the removal of clot from the ventricular system [120]. The phase III CLEAR-IVH III trial [121], comparing the use of EVD combined with intraventricular injection of r-tPA to EVD plus intraventricular injection of normal saline (placebo) for the treatment of IVH, has been completed (500 subjects enrolled from 73 sites between 2009 and 2014) and preliminary results have been presented recently at the International Stroke Conference (ISC) 2016. The primary outcome of dichotomized mRS $0-3$ vs $4-6$ at 180 days was not significantly different between the two groups, but the treatment was associated with a $10 \%$ reduction in mortality without increasing the number of patients in a vegetative state or with severe disability. The CLEAR-IVH III researchers also reported that patients with larger clots and more than $20 \mathrm{ml}$ of blood removed showed a significant improvement in functional outcome. In terms of safety, symptomatic bleeding was not more frequent in the alteplase group, and it was associated with a reduction in bacterial ventriculitis $(7 \%$ vs $12 \%, p=0.05)$ (official publication of results awaited) [122]. Li et al. [123], in a systematic review and metaanalysis of 11 studies including five RCTs (680 patients), found that the neuroendoscopy + EVD approach seemed to be better than the EVD + IVT approach in terms of mortality, effective hematoma evacuation rate, good functional outcome, and the ventriculoperitoneal shunt dependence rate.

\section{Minimally invasive surgery}

New approaches for hematoma drainage have emerged in the last decade, including stereotactic aspiration of clot \pm thrombolysis or endoscopic procedures. Overall, minimally invasive surgery has been associated with improvement in clot removal compared with standard surgical techniques [124, 125]. Cho et al. [126] compared three approaches (neuroendoscopy vs stereotactic aspiration vs craniotomy) in a randomized trial of 90 noncomatose patients with ganglionic hematomas. There was no difference in mortality but patients treated endoscopically had better functional outcomes within 6 months of surgery as assessed by functional independence measure score, Barthel index score, and muscle power. A recent systematic review and meta-analysis showed that death or dependence is significant reduced by minimally invasive surgery when compared with medical management or conventional craniotomy [127]. The Minimally Invasive Surgery Plus rt-PA for ICH Evacuation Phase III (MISTIE III) trial (ClinicalTrials.gov NCT01827046) is currently assessing the usefulness of stereotactic catheter placement into intraparenchymal hematomas followed by direct injection of r-tPA for 3 days and aspiration.

\section{Quality in acute stroke care}

There is evidence for the benefit of stroke units [70] with a significant positive impact on long-term outcome (i.e., decreased morbidity and mortality). Medical complications such as fever, hyperglycemia, VTE, and dysphagia are still not universally optimally managed [128]. Hyperthermia (temperature $>37.5{ }^{\circ} \mathrm{C}$ ), hyperglycemia, and dysphagia occur in $20-50 \%$, up to $50 \%$, and between 37 and $78 \%$ of patients, in the first days after the stroke, respectively.

A. Hyperglycemia is common in patients presenting with ICH and is associated with poor outcomes (hematoma expansion, increased edema, death, or severe disability) [129-132]. The optimal glucose level and the best hyperglycemia management strategy remain to be elucidated. However, both hypoglycemia $(<70 \mathrm{mg} / \mathrm{dl}$ or $<3.9 \mathrm{mmol} / \mathrm{L})$ and hyperglycemia ( $>180 \mathrm{mg} / \mathrm{dl}$ or $10 \mathrm{mmol} / \mathrm{L}$ ) should be avoided [9].

B. Temperature. Fever is a common occurrence affecting between 30 and $50 \%$ of patients with $\mathrm{ICH}$, and is independently associated with poor outcomes $[133,134]$. The presence of IVH is the main risk factor for fever not explained by infections or drugs [135]. There are no available data from RCTs addressing the role of induced normothermia after $\mathrm{ICH}$. At this point, the suggested goal is to keep a core temperature below $37.5-38{ }^{\circ} \mathrm{C}$ [9]. One pilot study (iCOOL1) randomized 20 patients with acute stroke to induced normothermia (cold saline infusion $\left(4{ }^{\circ} \mathrm{C}, 2 \mathrm{~L}\right.$ at $4 \mathrm{~L} /$ hour $)$ vs nasopharyngeal cooling ( $60 \mathrm{~L} /$ minute for 1 hour)). A high incidence of serious adverse events (seven in total) was reported, suggesting that safety of cooling in acute stroke patients awaits evaluation in future trials [136].

C. Prevention of VTE. Patients with ICH are considered at high risk of VTE, reported to be up to 4fold higher than in patients with ischemic stroke [137]. Initial prophylaxis utilizes intermittent pneumatic compression devices to be positioned at the time of hospital admission (Strong recommendation and high-quality evidence) $[138,139]$, followed by pharmacological prophylaxis with UFH or LMWH, initiated after documented cessation of bleeding by imaging [140]. This is often considered to be $24-48$ hours from the initial hemorrhage [139].

D. Dysphagia is common after stroke, and its reported incidence varies from 37 to $78 \%$, depending on the technique used for detection [141]. Dysphagia is associated with increased risk for pneumonia/ 
pneumonitis (RR 3.17, 95 \% CI 2.07-4.87) [141]. Occurrence of aspiration pneumonia can be reduced by formal dysphagia screening (e.g., water swallow test) from $5.4 \%$ to $2.4 \%$ (3 \% absolute risk reduction) [142]. According to the AHA/ASA guidelines, "a formal screening procedure for dysphagia should be performed in all patients before the initiation of oral intake to reduce the risk of pneumonia" (Class I; Level of Evidence B) [9].

E. Anemia. Hemoglobin levels on admission and nadir levels seem to be associated with outcome after $\mathrm{ICH}$ $[143,144]$. However, there is currently no universally accepted ideal hemoglobin level in this population.

F. Seizure prophylaxis. Seizure frequency has been reported between 8.1 and $10.6 \%$ in patients with $\mathrm{ICH}$, with status epilepticus occurring in 1-2 \% of patients [9]. Lobar hemorrhages are independent predictor of early and late seizures (i.e., occurring more than 2 weeks after ictus). However, prophylactic use of anticonvulsants in $\mathrm{ICH}$ patients is associated with worse outcomes. Phenytoin has been associated with increased side effects and worse outcomes [145]. The current AHA ICH guidelines do not recommend the use of prophylactic anticonvulsants [9]. Continuous EEG has become a monitor that is used in the management of patients with $\mathrm{ICH}$. Claassen et al. [146] reviewed the records of 102 consecutive patients who underwent continuous EEG monitoring after ICH. They found that one-third of ICH patients developed seizures, with more than half having only electroencephalographic evidence of seizures. They also found a correlation between electroencephalographic seizures and expanding hemorrhages, and also between periodic discharges in patients with cortical ICH and poorer outcomes [146]. Patients with ICH presenting with a decreased level of consciousness out of proportion to the clinical expected level of neurologic impairment may benefit from continuous EEG monitoring, which can be used to trigger or modify therapy [9].

Management of increased ICP is a topic beyond the scope of this review, and we refer to very informative articles published recently [147]. In general, ICP management strategies include: head of bed elevation between 30 and $45^{\circ}$, CSF drainage through EVD, analgesia and sedation, normocapneic ventilation, and administration of hypertonic solutions (e.g., hypertonic saline or mannitol). In refractory cases, hypothermia, barbiturates, or decompressive craniectomy can be attempted [147].

\section{Challenges in prognostication}

Several clinical ICH grading scales have been published with the ultimate goal of risk stratification and prognostication [68]. However, a confounder in the accuracy of these scales is the impact of withholding or withdrawal of life support (WOLS). Most ICH patients die in the acute phase because of WOLS [148, 149]; especially in patients with a high ICH score, WOLS is associated with early death [148, 149]. Diringer et al. [150] showed in a large series of more than 2000 mechanically ventilated patients admitted to a neuroscience ICU that older or comatose patients were more likely to have their life support withdrawn. Because of the uncertainty in prognostication in the early phase, early aggressive management is recommended after $\mathrm{ICH}$, and treatment limitations should not be based solely on prognostic models [9]

\section{Conclusion}

Spontaneous ICH is a neurological emergency associated with high mortality and morbidity. Key management issues include prompt etiologic diagnosis, reversal of anticoagulation, consideration of surgical management, and control of blood pressure. The clinical scenario and local practice will influence choice of therapeutic environment. As of March 2016, there were 581 ClinicalTrials.gov registered trials evaluating therapeutic opportunities in $\mathrm{ICH}$. Early prognostication should not be attempted except when clear signs of nonconfounded irreversible brain damage are present, such as an absence of brain stem reflexes.

\section{Additional file}

Additional file 1: Table S1. Etiology of spontaneous ICH. Table S2. Initial management. Table S3. Composition of some four-factor PCCS. Table S4. Ongoing studies on ICH management, and Table S5. Hematoma expansion scores. (DOCX 28 kb)

\section{Abbreviations \\ AAICH, anticoagulant-associated intracerebral hemorrhage; APT, antiplatelet therapy; ATACH, Antihypertensive Treatment of Acute Cerebral Hemorrhage; $\mathrm{BP}$, blood pressure; $\mathrm{CBF}$, cerebral blood flow; $\mathrm{CMRO}_{2}$, cerebral metabolic rate of oxygen; CPP, cerebral perfusion pressure; CSF, cerebrospinal fluid; CT, computed tomography; CTA, computed tomography angiography; DSA, digital subtraction angiography; ED, emergency department; EVD, external ventricular drain; FFP, fresh frozen plasma; GCS, Glasgow Coma Scale; $\mathrm{ICH}$, intracerebral hemorrhage; ICP, intracranial pressure; INR, International Normalized Ratio; IVH, intraventricular hemorrhage; IVT, intraventricular thrombolysis; MRI, magnetic resonance imaging; mRS, modified Rankin Scale; NIHSS, National Institutes of Health Stroke Scale; OEF ${ }^{\max ,}$ maximum oxygen extraction fraction; OR, odds ratio; PCC, prothrombin complex concentrate; rFVlla, recombinant activated factor VIl; r-tPA, recombinant tissue plasminogen activator; SBP, systolic blood pressure; VTE, venous thromboembolism; WOLS, withdrawal of life support}

\section{Acknowledgements}

This work was supported by generous funding provided by the Bitove Foundation

\section{Authors' contributions}

DT-P, FGZ, AG, AD, SA, and ALdOM formed the conceptual framework for this article. AG and ALdOM conducted the literature search. ALdOM, FGZ, and $\mathrm{AG}$ were responsible for the tables. ALdOM and AG were responsible for the figures. TRM, AD, RLM, and SA provided critical support and gave 
important scientific feedback on the manuscript. All authors read and approved the final manuscript.

\section{Competing interests}

The authors declare that they have no competing interests.

\section{Author details}

'Department of Medical Imaging, Interventional Neuroradiology, St. Michael's Hospital_University of Toronto, 30 Bond Street, Toronto, ON M5B 1W8, Canada. ${ }^{2}$ Department of Critical Care Medicine, Trauma \& Neurosurgical Intensive Care Unit, St. Michael's Hospital—University of Toronto, Toronto, ON, Canada. ${ }^{3}$ Neuroscience Research Program, Keenan Research Centre for Biomedical Science of St. Michael's Hospital, Toronto, ON, Canada. ${ }^{4}$ Department of Medicine, Division of Respirology (Critical Care), University Health Network, Toronto, ON, Canada. ${ }^{5}$ nterdepartmental Division of Critical Care Medicine and Department of Medicine, University of Toronto, Toronto, ON, Canada. ${ }^{6}$ Intensive Care Unit, Hospital das Clínicas, Universidade de São Paulo, São Paulo, Brazil. ${ }^{7}$ Intensive Care Unit, Hospital Alemão Oswaldo Cruz, São Paulo, Brazil. ${ }^{8}$ Medical Intensive Care Unit, Department of Pulmonary, Allergy, and Critical Care, Respiratory Institute, Cleveland Clinic, Cleveland, $\mathrm{OH}$, USA. ${ }^{9}$ Cleveland Clinic Lerner College of Medicine of Case Western Reserve University, Cleveland, OH, USA. ${ }^{10}$ Department of Surgery, Division of Neurosurgery, St. Michael's Hospital-University of Toronto, Toronto, ON, Canada. ${ }^{11}$ Department of Anesthesiology, St. Michael's Hospital_University of Toronto, Toronto, ON, Canada.

\section{Published online: 18 September 2016}

\section{References}

1. Xi G, Keep RF, Hoff JT. Mechanisms of brain injury after intracerebral haemorrhage. Lancet Neurol. 2006:5:53-63.

2. Zheng H, Chen C, Zhang J, Hu Z. Mechanism and Therapy of Brain Edema after Intracerebral Hemorrhage. Cerebrovasc Dis. 2016;42:155-169.

3. Qureshi Al, Mendelow AD, Hanley DF. Intracerebral haemorrhage. Lancet. 2009;373(9675):1632-44. doi:10.1016/S0140-6736(09)60371-8.

4. van Asch CJ, Luitse MJ, Rinkel GJ, van der Tweel I, Algra A, Klijn CJ. Incidence, case fatality, and functional outcome of intracerebral haemorrhage over time, according to age, sex, and ethnic origin: a systematic review and meta-analysis. Lancet Neurol. 2010;9(2):167-76. doi:10.1016/S1474-4422(09)70340-0.

5. Balami JS, Buchan AM. Complications of intracerebral haemorrhage. Lancet Neurol. 2011;11(1):101-18. doi:10.1016/S1474-4422(11)70264-2.

6. Krishnamurthi RV, Feigin VL, Forouzanfar MH, et al. Global and regional burden of first-ever ischaemic and haemorrhagic stroke during 19902010: findings from the Global Burden of Disease Study 2010. Lancet Glob Health. 2013;1(5):e259-81. doi:10.1016/S2214-109X(13)70089-5.

7. Krishnamurthi RV, Moran AE, Forouzanfar $\mathrm{MH}$, et al. The global burden of hemorrhagic stroke. Global Heart. 2014;9(1):101-6. doi:10.1016/j.gheart.2014.01.003.

8. Sacco S, Marini C, Toni D, Olivieri L, Carolei A. Incidence and 10-year survival of intracerebral hemorrhage in a population-based registry. Stroke. 2009:40(2):394-9. doi:10.1161/STROKEAHA.108.523209.

9. Hemphill JC, Greenberg SM, Anderson CS, et al. Guidelines for the Management of Spontaneous Intracerebral Hemorrhage: A Guideline for Healthcare Professionals from the American Heart Association/American Stroke Association. Stroke. 2015;46(7):2032-60. doi:10.1161/STR. 0000000000000069.

10. Rincon F, Mayer SA. The epidemiology of intracerebral hemorrhage in the United States from 1979 to 2008. Neurocrit Care. 2012;19(1):95-102. doi:10.1007/s12028-012-9793-y.

11. Becker KJ, Baxter AB, Cohen WA, et al. Withdrawal of support in intracerebral hemorrhage may lead to self-fulfilling prophecies. Neurology. 2001;56(6):766-72.

12. Gattellari M, Goumas C, Worthington J. Declining rates of fatal and nonfatal intracerebral hemorrhage: epidemiological trends in Australia. J Am Heart Assoc. 2014;3(6):e001161. doi:10.1161/JAHA.114.001161.

13. Koton S, Schneider ALC, Rosamond WD, et al. Stroke incidence and mortality trends in US communities, 1987 to 2011. JAMA. 2014;312(3):259-68. doi:10.1001/jama.2014.7692.

14. Flaherty ML, Kissela B, Woo D, et al. The increasing incidence of anticoagulantassociated intracerebral hemorrhage. Neurology. 2007;68(2):116-21. doi:10.1212/01.wnl.0000250340.05202.8b.
15. Miller CS, Grandi SM, Shimony A, Filion KB, Eisenberg MJ. Meta-analysis of efficacy and safety of new oral anticoagulants (dabigatran, rivaroxaban, apixaban) versus warfarin in patients with atrial fibrillation. Am J Cardiol. 2012;110(3):453-60. doi:10.1016/j.amjcard.2012.03.049.

16. Abu-Zeid HAH. Prognostic factors in the survival of 1,484 stroke cases observed for 30 to 48 months. Arch Neurol. 1978;35(3):121. doi:10.1001/ archneur.1978.00500270003001.

17. Carpenter AM, Singh IP, Gandhi CD, Prestigiacomo CJ. Genetic risk factors for spontaneous intracerebral haemorrhage. Nat Publ Group. 2015;12(1):40-9. doi:10.1038/nrneurol.2015.226.

18. Martini SR, Flaherty ML, Brown WM, et al. Risk factors for intracerebral hemorrhage differ according to hemorrhage location. Neurology. 2013;79(23):2275-82. doi:10.1212/WNL.0b013e318276896f.

19. Flaherty ML, Woo D, Haverbusch M, et al. Racial variations in location and risk of intracerebral hemorrhage. Stroke. 2005;36(5):934-7. doi:10.1161/01. STR.0000160756.72109.95.

20. Ariesen MJ, Claus SP, Rinkel GJE, Algra A. Risk factors for intracerebral hemorrhage in the general population: a systematic review. Stroke. 2003;34(8):2060-5. doi:10.1161/01.STR.0000080678.09344.8D.

21. Sturgeon JD, Folsom AR, Longstreth WT, Shahar E, Rosamond WD, Cushman M. Risk factors for intracerebral hemorrhage in a pooled prospective study. Stroke. 2007;38(10):2718-25. doi:10.1161/STROKEAHA.107.487090.

22. Yamada M. Cerebral amyloid angiopathy: emerging concepts. J Stroke. 2015;17(1):17-30. doi:10.5853/jos.2015.17.1.17.

23. McCarron MO, Nicoll JA. Apolipoprotein E genotype and cerebral amyloid angiopathy-related hemorrhage. Ann N Y Acad Sci. 2000;903:176-9.

24. Flibotte JJ, Hagan N, O'Donnell J, Greenberg SM, Rosand J. Warfarin hematoma expansion, and outcome of intracerebral hemorrhage. Neurology. 2004;63(6):1059-64.

25. Howard G, Cushman M, Howard VJ, et al. Risk factors for intracerebral hemorrhage: the REasons for geographic and racial differences in stroke (REGARDS) study. Stroke. 2013;44(5):1282-7. doi:10.1161/STROKEAHA.111.000529.

26. Jauch EC, Pineda JA, Claude HJ. Emergency neurological life support: intracerebral hemorrhage. Neurocrit Care. 2015;23 Suppl 2:83-93. doi:10.1007/s12028-015-0167-0.

27. Maas MB, Berman MD, Guth JC, Liotta EM, Prabhakaran S, Naidech AM. Neurochecks as a biomarker of the temporal profile and clinical impact of neurologic changes after intracerebral hemorrhage. J Stroke Cerebrovasc Dis. 2015;24(9):2026-31. doi:10.1016/j.jstrokecerebrovasdis.2015.04.045.

28. Maas MB, Rosenberg NF, Kosteva AR, et al. Surveillance neuroimaging and neurologic examinations affect care for intracerebral hemorrhage. Neurology. 2013;81(2):107-12. doi:10.1212/WNL.0b013e31829a33e4.

29. Runchey S, McGee S. Does this patient have a hemorrhagic stroke?: clinical findings distinguishing hemorrhagic stroke from ischemic stroke. JAMA. 2010;303(22):2280-6. doi:10.1001/jama.2010.754.

30. Alobeidi F, Aviv RI. Emergency imaging of intracerebral haemorrhage. Front Neurol Neurosci. 2015;37:13-26. doi:10.1159/000437110.

31. Zhu XLX, Chan MSM, Poon WSW. Spontaneous intracranial hemorrhage: which patients need diagnostic cerebral angiography? A prospective study of 206 cases and review of the literature. Stroke. 1997;28(7):1406-9. doi:10.1161/01.STR.28.7.1406.

32. Bekelis K, Desai A, Zhao W, et al. Computed tomography angiography: improving diagnostic yield and cost effectiveness in the initial evaluation of spontaneous nonsubarachnoid intracerebral hemorrhage. J Neurosurg. 2012;117(4):761-6. doi:10.3171/2012.7.JNS12281.

33. Stam J. Thrombosis of the cerebral veins and sinuses. N Engl J Med. 2005;352(17):1791-8. doi:10.1056/NEJMra042354.

34. Delgado Almandoz JE, Schaefer PW, Forero NP, Falla JR, Gonzalez RG, Romero JM. Diagnostic accuracy and yield of multidetector CT angiography in the evaluation of spontaneous intraparenchymal cerebral hemorrhage. Am J Neuroradiol. 2009;30(6):1213-21. doi:10.3174/ajnr.A1546.

35. Yeung R, Ahmad T, Aviv Rl, de Tilly LN, Fox AJ, Symons SP. Comparison of CTA to DSA in determining the etiology of spontaneous ICH. Can J Neurol Sci. 2009; 36(2):176-80.

36. Wong GKC, Siu DYW, Abrigo JM, et al. Computed tomographic angiography and venography for young or nonhypertensive patients with acute spontaneous intracerebral hemorrhage. Stroke. 2011;42(1):211-3. doi:10.1161/STROKEAHA.110.592337.

37. Broderick JP, Brott TG, Duldner JE, Tomsick T, Huster G. Volume of intracerebral hemorrhage. A powerful and easy-to-use predictor of 30-day mortality. Stroke. 1993;24(7):987-93. 
38. Hemphill JC, Bonovich DC, Besmertis L, Manley GT, Johnston SC, Tuhrim S. The ICH score: a simple, reliable grading scale for intracerebral hemorrhage. Stroke. 2001;32(4):891-7. doi:10.1161/01.STR.32.4.891.

39. Webb AJS, Ullman NL, Morgan TC, et al. Accuracy of the ABC/2 score for intracerebral hemorrhage: systematic review and analysis of MISTIE, CLEAR-IVH, and CLEAR III. Stroke. 2015;46(9):2470-6. doi:10.1161/STROKEAHA.114.007343.

40. Kothari RU, Brott T, Broderick JP, et al. The ABCs of measuring intracerebral hemorrhage volumes. Stroke. 1996;27(8):1304-5. doi:10.1161/01.STR.27.8.1304.

41. Hallevi H, Albright KC, Aronowski J, et al. Intraventricular hemorrhage: anatomic relationships and clinical implications. Neurology. 2008;70(11):848-52. doi:10.1212/01.wnl.0000304930.47751.75.

42. Bhattathiri PS, Gregson B, Prasad KSM, Mendelow AD, STICH Investigators. Intraventricular hemorrhage and hydrocephalus after spontaneous intracerebral hemorrhage: results from the STICH trial. Acta Neurochir Suppl. 2006;96:65-8.

43. Steiner T, Diringer MN, Schneider D, et al. Dynamics of intraventricular hemorrhage in patients with spontaneous intracerebral hemorrhage: risk factors, clinical impact, and effect of hemostatic therapy with recombinant activated factor VII. Neurosurgery. 2006;59(4):767-73. doi:10.1227/01.NEU. 0000232837.34992.32. discussion773-4.

44. Mayer SA, Sacco RL, Shi T, Mohr JP. Neurologic deterioration in noncomatose patients with supratentorial intracerebral hemorrhage. Neurology. 1994;44(8):1379-84.

45. Brott $T$, Broderick J, Kothari R, et al. Early hemorrhage growth in patients with intracerebral hemorrhage. Stroke. 1997;28(1):1-5. doi:10.1161/01.STR.28.1.1.

46. Specogna AV, Turin TC, Patten SB, Hill MD. Factors associated with early deterioration after spontaneous intracerebral hemorrhage: a systematic review and meta-analysis. PLoS One. 2014:9(5):e96743-3. doi:10.1371/journal.pone.0096743.

47. Davis SM, Broderick J, Hennerici M, et al. Hematoma growth is a determinant of mortality and poor outcome after intracerebral hemorrhage. Neurology. 2006:66(8):1175-81. doi:10.1212/01.wnl.0000208408.98482.99.

48. Dowlatshahi D, Demchuk AM, Flaherty ML, et al. Defining hematoma expansion in intracerebral hemorrhage: relationship with patient outcomes. Neurology. 2011;76(14):1238-44. doi:10.1212/WNL.0b013e3182143317.

49. Kazui S, Naritomi H, Yamamoto H, Sawada T, Yamaguchi T. Enlargement of spontaneous intracerebral hemorrhage. Incidence and time course. Stroke. 1996;27(10):1783-7

50. Brouwers HB, Chang Y, Falcone GJ, et al. Predicting hematoma expansion after primary intracerebral hemorrhage. JAMA Neurol. 2014;71(2):158-64. doi:10.1001/jamaneurol.2013.5433.

51. Wang X, Arima H, Al-Shahi Salman R, et al. Clinical prediction algorithm (BRAIN) to determine risk of hematoma growth in acute intracerebral hemorrhage. Stroke. 2015;46(2):376-81. doi:10.1161/STROKEAHA.114.006910.

52. Huynh TJ, Aviv RI, Dowlatshahi D, Gladstone DJ. Validation of the 9-point and 24-point hematoma expansion prediction scores and derivation of the PREDICT A/B scores. Stroke. 2015. doi:10.1161/STROKEAHA.115.009893/-/DC1.

53. Du F-Z, Jiang R, Gu M, He C, Guan J. The accuracy of spot sign in predicting hematoma expansion after intracerebral hemorrhage: a systematic review and meta-analysis. PLoS One. 2014;9(12):e115777-7. doi:10.1371/journal. pone.0115777.

54. Becker KJ, Baxter AB, Bybee HM, Tirschwell DL, Abouelsaad T, Cohen WA Extravasation of radiographic contrast is an independent predictor of death in primary intracerebral hemorrhage. Stroke. 1999;30(10):2025-32.

55. Murai $Y$, Takagi $R$, lkeda $Y$, Yamamoto $Y$, Teramoto A. Three-dimensional computerized tomography angiography in patients with hyperacute intracerebral hemorrhage. J Neurosurg. 1999:91(3):424-31. doi:10.3171/jns.1999.91.3.0424.

56. Goldstein JN, Fazen LE, Snider R, et al. Contrast extravasation on CT angiography predicts hematoma expansion in intracerebral hemorrhage. Neurology. 2007;68(12):889-94. doi:10.1212/01.wnl.0000257087.22852.21.

57. Wada R, Aviv RI, Fox AJ, et al. CT Angiography "spot sign" predicts hematoma expansion in acute intracerebral hemorrhage. Stroke. 2007:38(4):1257-62. doi:10.1161/01.STR.0000259633.59404.f3.

58. Ederies A, Demchuk A, Chia T, et al. Postcontrast CT extravasation is associated with hematoma expansion in CTA spot negative patients. Stroke. 2009:40(5):1672-6. doi:10.1161/STROKEAHA.108.541201.

59. Hallevi H, Abraham AT, Barreto AD, Grotta JC, Savitz SI. The spot sign in intracerebral hemorrhage: the importance of looking for contrast extravasation. Cerebrovasc Dis. 2010;29(3):217-20. doi:10.1159/000267842.

60. Brouwers HB, Goldstein JN, Romero JM, Rosand J. Clinical applications of the computed tomography angiography spot sign in acute intracerebral hemorrhage: a review. Stroke. 2012;43(12):3427-32. doi:10.1161/STROKEAHA.112.664003.
61. Tsukabe A, Watanabe $\mathrm{Y}$, Tanaka $\mathrm{H}$, et al. Prevalence and diagnostic performance of computed tomography angiography spot sign for intracerebral hematoma expansion depend on scan timing. Neuroradiology. 2014:56(12):1039-45. doi:10.1007/s00234-014-1430-3.

62. Almandoz JED, Yoo AJ, Stone MJ, et al. Systematic characterization of the computed tomography angiography spot sign in primary intracerebral hemorrhage identifies patients at highest risk for hematoma expansion: the spot sign score. Stroke. 2009;40(9):2994-3000. doi:10.1161/ STROKEAHA.109.554667.

63. Delgado Almandoz JE, Yoo AJ, Stone MJ, et al. The spot sign score in primary intracerebral hemorrhage identifies patients at highest risk of in-hospital mortality and poor outcome among survivors. Stroke. 2009:41(1):54-60. doi:10.1161/STROKEAHA.109.565382.

64. Rodriguez-Luna D, Dowlatshahi D, Aviv Rl, et al. Venous phase of computed tomography angiography increases spot sign detection, but intracerebral hemorrhage expansion is greater in spot signs detected in arterial phase. Stroke. 2014:45(3):734-9. doi:10.1161/STROKEAHA.113.003007.

65. Evans A, Demchuk A, Symons SP, et al. The spot sign is more common in the absence of multiple prior microbleeds. Stroke. 2010;41(10):2210-7. doi:10.1161/STROKEAHA.110.593970.

66. Demchuk AM, Dowlatshahi D, Rodriguez-Luna D. Prediction of haematoma growth and outcome in patients with intracerebral haemorrhage using the CT-angiography spot sign (PREDICT): a prospective observational study. Lancet Neurol. 2012;11(4):307-14. doi:10.1016/s1474-4422(12)70038-8.

67. Goldstein JN, Brouwers HB, Romero JM, et al. SCORE-IT: the Spot Sign score in restricting ICH growth—an Atach-II ancillary study. J Vasc Interv Neurol. 2012;5 Suppl:20.

68. Hwang BY, Appelboom G, Kellner CP, et al. Clinical grading scales in intracerebral hemorrhage. Neurocrit Care. 2010;13(1):141-51. doi:10.1007/ s12028-010-9382-x.

69. Diringer MN, Edwards DF. Admission to a neurologic/neurosurgical intensive care unit is associated with reduced mortality rate after intracerebral hemorrhage. Crit Care Med. 2001;29(3):635-40.

70. Stroke Unit Trialists' Collaboration. Organised inpatient (stroke unit) care for stroke. Cochrane Database Syst Rev. 2013;9:CD000197. doi:10.1002/14651858.CD000197.pub3.

71. Anderson CS, Heeley E, Huang Y, et al. Rapid blood-pressure lowering in patients with acute intracerebral hemorrhage. N Engl J Med. 2013;368(25):2355-65. doi:10.1056/NEJMoa1214609.

72. Terént A, Asplund K, Farahmand B, et al. Stroke unit care revisited: who benefits the most? A cohort study of 105,043 patients in Riks-Stroke, the Swedish Stroke Register. J Neurol Neurosurg Psychiatry. 2009;80(8):881-7. doi:10.1136/jnnp.2008.169102.

73. Zazulia ARA, Diringer MNM, Videen TOT, et al. Hypoperfusion without ischemia surrounding acute intracerebral hemorrhage. J Cereb Blood Flow Metab. 2001;21(7):804-10. doi:10.1097/00004647-200107000-00005.

74. Butcher KS, Jeerakathil T, Hill M, et al. The Intracerebral Hemorrhage Acutely Decreasing Arterial Pressure Trial. Stroke. 2013;44(3):620-6. doi:10.1161/STROKEAHA.111.000188.

75. Kate MP, Hansen MB, Mouridsen K, et al. Blood pressure reduction does not reduce perihematoma oxygenation: a CT perfusion study. J Cereb Blood Flow Metab. 2013;34(1):81-6. doi:10.1038/jcbfm.2013.164.

76. Anderson CS, Huang Y, Wang JG, et al. Intensive blood pressure reduction in acute cerebral haemorrhage trial (INTERACT): a randomised pilot trial. Lancet Neurol. 2008;7(5):391-9. doi:10.1016/S1474-4422(08)70069-3.

77. Qureshi Al, Palesch YY, Barsan WG, et al. Intensive Blood-Pressure Lowering in Patients with Acute Cerebral Hemorrhage. N Engl J Med. 2016. doi:10.1056/NEJMoa1603460. [Epub ahead of print]

78. Prabhakaran S, Naidech AM. Ischemic brain injury after intracerebral hemorrhage: a critical review. Stroke. 2012;43(8):2258-63. doi:10.1161/ STROKEAHA.112.655910.

79. Ortega-Gutierrez S, Thomas J, Reccius A, et al. Effectiveness and safety of nicardipine and labetalol infusion for blood pressure management in patients with intracerebral and subarachnoid hemorrhage. Neurocrit Care. 2013;18(1):13-9. doi:10.1007/s12028-012-9782-1.

80. Liu-DeRyke X, Levy PD, Parker D, Coplin W, Rhoney DH. A prospective evaluation of labetalol versus nicardipine for blood pressure management in patients with acute stroke. Neurocrit Care. 2013;19(1):41-7. doi:10.1007/ s12028-013-9863-9.

81. Rosand J, Eckman MH, Knudsen KA, Singer DE, Greenberg SM. The effect of warfarin and intensity of anticoagulation on outcome of 
intracerebral hemorrhage. Arch Intern Med. 2004;164(8):880-4. doi:10.1001/archinte.164.8.880.

82. Hart RG, Boop BS, Anderson DC. Oral Anticoagulants and intracranial hemorrhage: facts and hypotheses. Stroke. 1995;26(8):1471-7. doi:10.1161/01.STR.26.8.1471.

83. Steiner T, Rosand J, Diringer M. Intracerebral hemorrhage associated with oral anticoagulant therapy: current practices and unresolved questions. Stroke. 2006;37(1):256-62. doi:10.1161/01.STR.0000196989.09900.f8.

84. Wintzen AR, de Jonge $H$, Loeliger EA, Bots GT. The risk of intracerebral hemorrhage during oral anticoagulant treatment: a population study. Ann Neurol. 1984;16(5):553-8. doi:10.1002/ana.410160505.

85. Hylek EM. Risk factors for intracranial hemorrhage in outpatients taking warfarin. Ann Intern Med. 1994;120(11):897-902. doi:10.7326/0003-4819-120-11199406010-00001.

86. Hansen ML, Sorensen R, Clausen MT, et al. Risk of bleeding with single, dual, or triple therapy with warfarin, aspirin, and clopidogrel in patients with atrial fibrillation. Arch Intern Med. 2010;170:1433-41.

87. Pisters R, Lane DA, Nieuwlaat R, de Vos CB, Crijns HJGM, Lip GYH. A novel userfriendly score (HAS-BLED) to assess 1-year risk of major bleeding in patients with atrial fibrillation. Chest. 2010;138(5):1093-100. doi:10.1378/chest.10-0134.

88. Flaherty ML, Tao H, Haverbusch M, et al. Warfarin use leads to larger intracerebral hematomas. Neurology. 2008;71(14):1084-9. doi:10.1212/01. wnl.0000326895.58992.27.

89. Flaherty ML, Haverbusch M, Sekar P, et al. Location and outcome of anticoagulant-associated intracerebral hemorrhage. Neurocrit Care. 2006:5(3):197-201. doi:10.1385/NCC:5:3:197.

90. Frontera JA, lii JJL, Rabinstein AA, et al. Guideline for Reversal of Antithrombotics in Intracranial Hemorrhage: A Statement for Healthcare Professionals from the Neurocritical Care Society and Society of Critical Care Medicine. Neurocrit Care. 2016;24(1):6-46. doi:10.1007/s12028-0150222-X.

91. Vigué B, Ract C, Tremey B, et al. Ultra-rapid management of oral anticoagulant therapy-related surgical intracranial hemorrhage. Intensive Care Med. 2007;33(4):721-5. doi:10.1007/s00134-007-0528-z.

92. Voils SA, Baird B. Systematic review: 3-factor versus 4-factor prothrombin complex concentrate for warfarin reversal: does it matter? Thromb Res. 2012;130(6):833-40. doi:10.1016/j.thromres.2012.10.001.

93. Holland L, Warkentin TE, Refaai M, Crowther MA, Johnston MA, Sarode R. Suboptimal effect of a three-factor prothrombin complex concentrate (Profilnine-SD) in correcting supratherapeutic international normalized ratio due to warfarin overdose. Transfusion. 2009;49(6):1171-7. doi:10.1111/j.1537-2995.2008.02080.x.

94. Tazarourte K, Riou B, Tremey B, Samama C-M, Vicaut E, Vigué B. Guidelineconcordant administration of prothrombin complex concentrate and vitamin $\mathrm{K}$ is associated with decreased mortality in patients with severe bleeding under vitamin K antagonist treatment (EPAHK study). Crit Care. 2014;18(2):R81-1. doi:10.1186/cc13843.

95. Pernod G, Godiér A, Gozalo C, Tremey B, Sié P. French clinical practice guidelines on the management of patients on vitamin $\mathrm{K}$ antagonists in at-risk situations (overdose, risk of bleeding, and active bleeding). Thromb Res. 2010;126(3):e167-74. doi:10.1016/j.thromres.2010.06.017.

96. Hickey M, Gatien M, Taljaard M, Aujnarain A, Giulivi A, Perry JJ. Outcomes of urgent warfarin reversal with frozen plasma versus prothrombin complex concentrate in the emergency department. Circulation. 2013;128(4):360-4. doi:10.1161/CIRCULATIONAHA.113.001875.

97. Sarode R, Milling TJ, Refaai MA, et al. Efficacy and safety of a 4-factor prothrombin complex concentrate in patients on vitamin $\mathrm{K}$ antagonists presenting with major bleeding: a randomized, plasma-controlled, phase Illb study. Circulation. 2013;128(11):1234-43. doi:10.1161/ CIRCULATIONAHA. 113.002283

98. Ray B, Keyrouz SG. Management of anticoagulant-related intracranial hemorrhage: an evidence-based review. Crit Care. 2014;18(3):223-3. doi:10.1186/cc13889

99. Roquer J, Rodríguez Campello A, Gomis M, Ois A, Puente V, Munteis E. Previous antiplatelet therapy is an independent predictor of 30-day mortality after spontaneous supratentorial intracerebral hemorrhage. J Neurol. 2005;252(4):412-6. doi:10.1007/s00415-005-0659-5.

100. Saloheimo P, Ahonen M, Juvela S, Pyhtinen J, Savolainen E-R, Hillbom M. Regular aspirin-use preceding the onset of primary intracerebral hemorrhage is an independent predictor for death. Stroke. 2006;37(1):129-33. doi:10.1161/01.STR.0000196991.03618.31.
101. Toyoda K, Okada Y, Minematsu K, et al. Antiplatelet therapy contributes to acute deterioration of intracerebral hemorrhage. Neurology. 2005;65(7):1000-4. doi:10.1212/01.wnl.0000179178.37713.69.

102. Sansing LH, Messe SR, Cucchiara BL, Cohen SN, Lyden PD, Kasner SE. Prior antiplatelet use does not affect hemorrhage growth or outcome after ICH. Neurology. 2009;72(16):1397-402. doi:10.1212/01.wnl.0000342709.31341.88.

103. Moussouttas M, Malhotra R, Fernandez L, et al. Role of antiplatelet agents in hematoma expansion during the acute period of intracerebral hemorrhage. Neurocrit Care. 2009;12(1):24-9. doi:10.1007/s12028-0099290-0.

104. Caso V, Paciaroni M, Venti M, et al. Effect of on-admission antiplatelet treatment on patients with cerebral hemorrhage. Cerebrovasc Dis. 2007:24(2-3):215-8. doi:10.1159/000104480

105. Campbell PG, Sen A, Yadla S, Jabbour P, Jallo J. Emergency reversal of antiplatelet agents in patients presenting with an intracranial hemorrhage: a clinical review. WNEU. 2010;74(2-3):279-85. doi:10.1016/j.wneu.2010.05.030.

106. Naidech AM, Liebling SM, Rosenberg NF, et al. Early platelet transfusion improves platelet activity and may improve outcomes after intracerebral hemorrhage. Neurocrit Care. 2012;16(1):82-7. doi:10.1007/s12028-011-9619-3.

107. Baharoglu MI, Cordonnier C, Salman RA-S, et al. Platelet transfusion versus standard care after acute stroke due to spontaneous cerebral haemorrhage associated with antiplatelet therapy (PATCH): a randomised, open-label, phase 3 trial. Lancet. 2016. doi:10.1016/S0140-6736(16)30392-0.

108. Frontera JA, Lewin III JJ, Rabinstein AA, et al. Guideline for reversal of antithrombotics in intracranial hemorrhage. Neurocrit Care. 2015;24(1):6-46. doi:10.1007/s12028-015-0222-x

109. Naidech AM, Maas MB, Levasseur-Franklin KE, et al. Desmopressin improves platelet activity in acute intracerebral hemorrhage. Stroke. 2014;45(8):2451-3. doi:10.1161/STROKEAHA.114.006061.

110. Kapapa T, Röhrer S, Struve S, et al. Desmopressin acetate in intracranial haemorrhage. Neurol Res Int. 2014;2014:298767-7. doi:10.1155/2014/298767.

111. Tanaka-Esposito C, Chung MK. Selecting antithrombotic therapy for patients with atrial fibrillation. Cleve Clin J Med. 2014;82(1):49-63. doi:10.3949/ccjm.82a.140002.

112. Pollack Jr CV, Reilly PA, Eikelboom J, et al. Idarucizumab for dabigatran reversal. N Engl J Med. 2015;373(6):511-20. doi:10.1056/NEJMoa1502000.

113. Yuan ZH, Jiang JK, Huang WD, Pan J, Zhu JY, Wang JZ. A meta-analysis of the efficacy and safety of recombinant activated factor VII for patients with acute intracerebral hemorrhage without hemophilia. J Clin Neurosci. 2010;17(6):685-93. doi:10.1016/j.jocn.2009.11.020.

114. Mendelow AD, Gregson BA, Fernandes HM, et al. Early surgery versus initial conservative treatment in patients with spontaneous supratentorial intracerebral haematomas in the International Surgical Trial in Intracerebral Haemorrhage (STICH): a randomised trial. Lancet. 2005;365:387-97.

115. Mendelow AD, Gregson BA, Rowan EN, et al. Early surgery versus initial conservative treatment in patients with spontaneous supratentorial lobar intracerebral haematomas (STICH II): a randomised trial. Lancet. 2013;382(9890):397-408. doi:10.1016/S0140-6736(13)60986-1.

116. Kobayashi SS, Sato AA, Kageyama YY, Nakamura HH, Watanabe YY, Yamaura AA. Treatment of hypertensive cerebellar hemorrhage—surgical or conservative management? Neurosurgery. 1994;34(2):246-1.

117. Kirollos RW, Tyagi AK, Ross SA, van Hille PT, Marks PV. Management of spontaneous cerebellar hematomas: a prospective treatment protocol. Neurosurgery. 2001;49(6):1378-7.

118. Mathew P, Teasdale G, Bannan A, Oluoch-Olunya D. Neurosurgical management of cerebellar haematoma and infarct. J Neurol Neurosurg Psychiatry. 1995;59(3):287-92.

119. Raco A, Caroli E, Isidori A, Vangelista T, Salvati M. Management of acute cerebellar infarction: one institution's experience. Neurosurgery. 2003;53(5):1061-6. doi:10.1227/01.NEU.0000088766.34559.3E.

120. Webb AJS, Ullman NL, Mann S, Muschelli J, Awad IA, Hanley DF. Resolution of intraventricular hemorrhage varies by ventricular region and dose of intraventricular thrombolytic: The Clot Lysis: Evaluating Accelerated Resolution of IVH (CLEAR IVH) program. Stroke. 2012;43(6):1666-8. doi:10.1161/STROKEAHA.112.650523.

121. Ziai WC, Tuhrim S, Lane $K$, et al. A multicenter, randomized, double-blinded, placebo-controlled phase III study of Clot Lysis Evaluation of Accelerated Resolution of Intraventricular Hemorrhage (CLEAR III). Int J Stroke. 2013;9(4):536-42. doi:10.1111/ijs.12097.

122. International Stroke Conference (ISC) 2016. Abstracts LB12 and LB13. Presented 18 Feb 2016. http://professional.heart.org/idc/groups/ahamah- 
public/@wcm/@sop/@scon/documents/downloadable/ucm_481854.pdf. 4 Aug 2016.

123. Li Y, Zhang $H$, Wang $X$, et al. Neuroendoscopic surgery versus external ventricular drainage alone or with intraventricular fibrinolysis for intraventricular hemorrhage secondary to spontaneous supratentorial hemorrhage: a systematic review and meta-analysis. Quinn TJ, ed. PLoS ONE. 2013;8(11):e80599. doi:10.1371/journal.pone.0080599.

124. Teernstra OPM, Evers SMAA, Lodder J, Leffers P, Franke CL, Blaauw G. Stereotactic treatment of intracerebral hematoma by means of a plasminogen activator: a multicenter randomized controlled trial (SICHPA). Stroke. 2003;34(4):968-74. doi:10.1161/01.STR.0000063367.52044.40.

125. Morgan T, Zuccarello M, Narayan R, Keyl P, Lane K, Hanley D. Preliminary findings of the Minimally-invasive Surgery plus rtPA for Intracerebral Hemorrhage Evacuation (MISTIE) clinical trial. Acta Neurochir Suppl. 2008;105:147-51.

126. Cho D-Y, Chen C-C, Chang C-S, Lee W-Y, Tso M. Endoscopic surgery for spontaneous basal ganglia hemorrhage: comparing endoscopic surgery, stereotactic aspiration, and craniotomy in noncomatose patients. Surg Neurol. 2006;65(6):547-55. doi:10.1016/j.surneu.2005.09.032. discussion555-6.

127. Zhou X, Chen J, Li Q, et al. Minimally invasive surgery for spontaneous supratentorial intracerebral hemorrhage: a meta-analysis of randomized controlled trials. Stroke. 2012:43(11):2923-30. doi:10.1161/STROKEAHA.112.667535.

128. Middleton S, McElduff $P$, Ward J, et al. Implementation of evidence-based treatment protocols to manage fever, hyperglycaemia, and swallowing dysfunction in acute stroke (QASC): a cluster randomised controlled trial. Lancet. 2011;378(9804):1699-706. doi:10.1016/S0140-6736(11)61485-2.

129. Fogelholm R, Murros K, Rissanen A, Avikainen S. Admission blood glucose and short term survival in primary intracerebral haemorrhage: a population based study. J Neurol Neurosurg Psychiatry. 2005;76(3):349-53. doi:10.1136/jnnp.2003.034819.

130. Kimura $K$, Iguchi $Y$, Inoue $T$, et al. Hyperglycemia independently increases the risk of early death in acute spontaneous intracerebral hemorrhage. J Neurol Sci. 2007;255(1):90-4. doi:10.1016/j.jns.2007.02.005.

131. Godoy DA, Piñero GR, Svampa S, Papa F, Di Napoli M. Hyperglycemia and short-term outcome in patients with spontaneous intracerebral hemorrhage. Neurocrit Care. 2008;9(2):217-29. doi:10.1007/s12028-008-9063-1.

132. Tan X, He J, Li L, et al. Early hyperglycaemia and the early-term death in patients with spontaneous intracerebral haemorrhage: a meta-analysis. Intern Med J. 2014;44(3):254-60. doi:10.1111/imj.12352.

133. Rincon F, Lyden P, Mayer SA. Relationship between temperature, hematoma growth, and functional outcome after intracerebral hemorrhage. Neurocrit Care. 2013;18(1):45-53. doi:10.1007/s12028-012-9779-9.

134. Schwarz S, Häfner K, Aschoff A, Schwab S. Incidence and prognostic significance of fever following intracerebral hemorrhage. Neurology. 2000:54(2):354-61.

135. Commichau C, Scarmeas N, Mayer SA. Risk factors for fever in the neurologic intensive care unit. Neurology. 2003;60(5):837-41.

136. Poli S, Purrucker J, Priglinger M, et al. Rapid Induction of COOLing in Stroke Patients (iCOOL1): a randomised pilot study comparing cold infusions with nasopharyngeal cooling. Crit Care. 2014;18(5):582-2. doi:10.1186/s13054-014-0582-1.

137. Raslan AM, Fields JD, Bhardwaj A. Prophylaxis for venous thrombo-embolism in neurocritical care: a critical appraisal. Neurocrit Care. 2009;12(2):297-309. doi:10.1007/s12028-009-9316-7.

138. CLOTS (Clots in Legs Or sTockings after Stroke) Trials Collaboration, Dennis M, Sandercock $P$, et al. Effectiveness of intermittent pneumatic compression in reduction of risk of deep vein thrombosis in patients who have had a stroke (CLOTS 3): a multicentre randomised controlled trial. Lancet. 2013;382:516-24. doi:10.1016/50140-6736(13)61050-8.

139. Nyquist $P$, Bautista $C$, Jichici $D$, et al. Prophylaxis of venous thrombosis in neurocritical care patients: an evidence-based guideline: A Statement for Healthcare Professionals from the Neurocritical Care Society. Neurocrit Care. 2016;24(1):47-60. doi:10.1007/s12028-015-0221-y.

140. Lacut K, Bressollette L, Le Gal G, et al. Prevention of venous thrombosis in patients with acute intracerebral hemorrhage. Neurology. 2005;65(6):865-9. doi:10.1212/01.wnl.0000176073.80532.a2.

141. Martino R, Foley N, Bhogal S, Diamant N, Speechley M, Teasell R. Dysphagia after stroke: incidence, diagnosis, and pulmonary complications. Stroke. 2005:36(12):2756-63. doi:10.1161/01.STR.0000190056.76543.eb.

142. Hinchey JA, Shephard T, Furie K, et al. Formal dysphagia screening protocols prevent pneumonia. Stroke. 2005;36(9):1972-6. doi:10.1161/01.STR 0000177529.86868.8d.
143. Kuramatsu JB, Gerner ST, Lucking H, et al. Anemia is an independent prognostic factor in intracerebral hemorrhage: an observational cohort study. Crit Care. 2013;17(4):R148. doi:10.1186/cc12827.

144. Chang TR, Boehme AK, Aysenne A, et al. Nadir hemoglobin is associated with poor outcome from intracerebral hemorrhage. Springerplus. 2013;2:379-9. doi:10.1186/2193-1801-2-379.

145. Naidech AM, Garg RK, Liebling S, et al. Anticonvulsant use and outcomes after intracerebral hemorrhage. Stroke. 2009;40(12):3810-5. doi:10.1161/ STROKEAHA.109.559948.

146. Claassen J, Jetté N, Chum F, et al. Electrographic seizures and periodic discharges after intracerebral hemorrhage. Neurology. 2007;69(13):1356-65. doi:10.1212/01.wnl.0000281664.02615.6c

147. Stevens RD, Shoykhet M, Cadena R. Emergency neurological life support: intracranial hypertension and herniation. Neurocrit Care. 2015;23 Suppl 2:76-82. doi:10.1007/s12028-015-0168-z.

148. Zurasky JA, Aiyagari V, Zazulia AR, Shackelford A, Diringer MN. Early mortality following spontaneous intracerebral hemorrhage. Neurology. 2005;64(4):725-7. doi:10.1212/01.WNL.0000152045.56837.58.

149. Naidech AM, Bernstein RA, Bassin SL, et al. How patients die after intracerebral hemorrhage. Neurocrit Care. 2009;11(1):45-9. doi:10.1007/s12028-009-9186-z.

150. Diringer MNM, Edwards DFD, Aiyagari W, Hollingsworth HH. Factors associated with withdrawal of mechanical ventilation in a neurology/ neurosurgery intensive care unit. Crit Care Med. 2001;29(9):1792-7.

151. Wayangankar SA, Abu-Fadel MS, Aronow HD, et al. Hemorrhagic and ischemic outcomes after bivalirudin versus unfractionated heparin during carotid artery stenting: a propensity score analysis from the NCDR. Circ Cardiovasc Interv. 2013;6(2):131-8. doi:10.1161/CIRCINTERVENTIONS.112.974857.

152. Koch S, Romano JG, Forteza AM, Otero CM, Rabinstein AA. Rapid blood pressure reduction in acute intracerebral hemorrhage: feasibility and safety. Neurocrit Care. 2008;8(3):316-21. doi:10.1007/s12028-008-9085-8.

153. Mayer SA, Brun NC, Begtrup K, et al. Efficacy and safety of recombinant activated factor VII for acute intracerebral hemorrhage. N Engl J Med. 2008:358(20):2127-37. doi:10.1056/NEJMoa0707534.

154. Antihypertensive Treatment of Acute Cerebral Hemorrhage (ATACH) investigators. Antihypertensive treatment of acute cerebral hemorrhage. Crit Care Med. 2010;38(2):637-48. doi:10.1097/CCM.0b013e3181b9e1a5.

155. Tsivgoulis G, Katsanos AH, Butcher KS, et al. Intensive blood pressure reduction in acute intracerebral hemorrhage: a meta-analysis. Neurolog. 2014;83(17):1523-9. doi:10.1212/WNL.0000000000000917. 